\title{
Characterization of the variability in the $\mathrm{O}+\mathrm{B}$ eclipsing binary HD 165246*
}

\author{
C. Johnston ${ }^{1,2} \dagger$, N. Aimar ${ }^{1,3}$, M. Abdul-Masih ${ }^{4,1}$, D. M. Bowman ${ }^{1}$, T. R. White ${ }^{5,6}$, \\ C. Hawcroft ${ }^{1}$, H. Sana ${ }^{1}$, S. Sekaran ${ }^{1}$, K. Dsilva ${ }^{1}$, A. Tkachenko ${ }^{1}$, C. Aerts ${ }^{1,2,7}$ \\ ${ }^{1}$ Institute of Astronomy, KU Leuven, Celestijnenlaan 200D, 3001 Leuven, Belgium \\ ${ }^{2}$ Department of Astrophysics, IMAPP, Radboud University Nijmegen, P. O. Box 9010, 6500 GL Nijmegen, the Netherlands \\ ${ }^{3}$ Observatoire de Paris, Université PSL, 5 Place Jules Janssen, 92195 Meudon, France \\ ${ }^{4}$ European Southern Observatory, Alonso de Córdova 3107, Vitacura, Casilla 19001, Santiago de Chile, Chile \\ ${ }^{5}$ Sydney Institute for Astronomy (SIfA), School of Physics, University of Sydney, NSW 2006, Australia \\ ${ }^{6}$ Stellar Astrophysics Centre, Department of Physics and Astronomy, Aarhus University, Ny Munkegade 120, DK-8000 Aarhus C, Denmark \\ ${ }^{7}$ Max Planck Institute for Astronomy, Koenigstuhl 17, 69117 Heidelberg, Germany
}

Accepted XXX. Received YYY; in original form ZZZ

\begin{abstract}
$\mathrm{O}$-stars are known to experience a wide range of variability mechanisms originating at both their surface and their near-core regions. Characterization and understanding of this variability and its potential causes are integral for evolutionary calculations. We use a new extensive high-resolution spectroscopic data set to characterize the variability observed in both the spectroscopic and space-based photometric observations of the O+B eclipsing binary HD 165246. We present an updated atmospheric and binary solution for the primary component, involving a high level of microturbulence $\left(13_{-1.3}^{+1.0} \mathrm{~km} \mathrm{~s}^{-1}\right)$ and a mass of $M_{1}=23.7_{-1.4}^{+1.1} \mathrm{M}_{\odot}$, placing it in a sparsely explored region of the Hertzsprung-Russell diagram. Furthermore, we deduce a rotational frequency of $0.690 \pm 0.003 \mathrm{~d}^{-1}$ from the combined photometric and line-profile variability, implying that the primary rotates at $40 \%$ of its critical Keplerian rotation rate. We discuss the potential explanations for the overall variability observed in this massive binary, and discuss its evolutionary context.
\end{abstract}

Key words: stars: massive - asteroseismology - stars: oscillations (including pulsations) binaries: eclipsing - stars: individual: HD 165246

\section{INTRODUCTION}

Despite the impact that massive stars $\left(M>9 \mathrm{M}_{\odot}\right)$ have on the dynamical and chemical evolution of their environment and host galaxy, the physics that determine their evolution are poorly calibrated in theoretical stellar structure and evolutionary models (Kippenhahn et al. 2012; Langer 2012). In particular, these uncalibrated physics propagate into population synthesis predictions, chemical evolutionary models, and gravitational-wave progenitor predictions. As such, calibrating the input physics that govern massive star evolution is an important goal of astrophysical research. However, due to the diversity of mechanisms that may or may not be active in a

*Based on observations obtained with the HERMES spectrograph on the Mercator telescope, which is supported by the Research Foundation - Flanders (FWO), Belgium, the Research Council of KU Leuven, Belgium, the Fonds National de la Recherche Scientifique (F.R.S.-FNRS), Belgium, the Royal Observatory of Belgium, the Observatoire de Genéve, Switzerland and the Thüringer Landessternwarte Tautenburg, Germany.

†E-mail: colecampbell.johnston@kuleuven.be given star, calibrating evolutionary models of massive stars can be difficult.

Massive stars exhibit a multitude of phenomena, including (in some cases extreme) wind-mass loss (e.g., Vink et al. 2011; Sander et al. 2019), rapid rotation (e.g., Maeder 2009; Ekström et al. 2012; de Mink et al. 2013; Abdul-Masih et al. 2019), magnetic fields (e.g., Wade et al. 2016; Buysschaert et al. 2017), as well as pulsations (e.g., Aerts et al. 2010; Handler 2013; Bowman 2020). Additionally, massive $\mathrm{O}$ - and B-stars are observed to have a high binary or multiplicity fraction (Kiminki \& Kobulnicky 2012; Sana et al. 2012, 2013, 2014; Aldoretta et al. 2015). Large scale spectroscopic surveys have attempted to populate the upper main-sequence (MS) region of the Hertzsprung-Russell diagram (HRD) in order to characterize the variability there, but face the challenge of disentangling multiple sources of variability (Simón-Díaz et al. 2017; Godart et al. 2017; Burssens et al. 2020). All of these variability mechanisms manifest spectroscopically and photometrically, with their observable effects being useful in constraining the physics responsible for them.

Stars with initial masses between approximately 8 and $25 \mathrm{M}_{\odot}$, and between 3 and $9 \mathrm{M}_{\odot}$ have been observed to pulsate in coherent 
pressure (p) and gravity (g) modes (Aerts et al. 2010) excited by the $\kappa$-mechanism (Pamyatnykh 1999), with their dominant restoring forces being the pressure force and the buoyancy force, respectively. Historically, these pulsators were difficult to study from the ground given the periods involved, however, long time-base, high duty-cycle space based missions have revealed some of these massive pulsators to be multi-periodic hybrid pulsators (De Cat et al. 2004; Briquet et al. 2007; Daszyńska-Daszkiewicz \& Walczak 2010; Szewczuk \& Daszyńska-Daszkiewicz 2017; Burssens et al. 2019). In addition to coherent pulsations excited via the $\kappa$-mechanism, OB stars are also observed to exhibit internal gravity waves (IGWs; Aerts \& Rogers 2015; Aerts et al. 2017; Bowman et al. 2019b,a), which are stochastically excited travelling buoyancy waves generated at the interface of the convective core and radiative envelope. Irrespective of the excitation mechanism, these waves cause both spectroscopic and photometric variability in observations (see e.g. Bowman et al. 2020). Additionally, the photometric signal associated with IGWs has been independently reproduced via both 2D (Ratnasingam et al. 2020; Horst et al. 2020) and 3D hydrodynamical simulations (Edelmann et al. 2019).

Whenever active, any of these mechanisms can substantially impact the evolution of a star and its resulting end product. To this end, characterization of the signals present in observations of massive stars is required before they can unambiguously be attributed to some given mechanism(s). As such, massive stars in eclipsing binaries represent some of the best opportunities to achieve this goal, as they provide model-independent estimates of fundamental stellar parameters such as mass and radius. However, the currently-known sample of massive eclipsing binaries with data sets that allow for such precise parameter determination is limited (Torres et al. 2010; Bonanos et al. 2011; Koumpia \& Bonanos 2012; Lohr et al. 2018; Mahy et al. 2020a,b). Even more so, there are fewer systems for which there exists extensive photometric and spectroscopic data sets to characterize the variety of variability.

In this paper, we investigate and characterize the intrinsic variability in the massive detached eclipsing O+B binary HD 165246. To do this, we utilise an extensive spectroscopic data set combined with high-precision space photometry. In Section 3 we determine an updated binary solution, and fundamental parameters for the system. Furthermore, in Section 3.3 we investigate the photometric variability present in the residual light curve after subtraction of the binary model. In Section 4, we introduce and analyse the new spectroscopic data set. Finally, in Section 5, we discuss the potential mechanisms behind the observed variability and briefly discuss the evolutionary context of the system, and present our conclusions in Section 6.

\section{THE TARGET HD 165246}

HD $165246(V=7.6, \mathrm{O} 8 \mathrm{~V})$ has been the focus of several dedicated studies and has been included as part of a larger sample in some spectroscopic surveys of massive stars. Mayer et al. (2013) made use of 13 FEROS spectra and 617 ASAS3 V-band observations to derive an initial orbital solution for this system, improving on the linear ephemeris deduced by Otero (2007). Mayer et al. (2013) determined a projected rotational velocity of $v \sin i=242.6 \pm 2.7 \mathrm{~km} \mathrm{~s}^{-1}$, without including micro- or macroturbulence. In their analysis, Mayer et al. (2013) assumed a mass for the primary of $M_{1}=21.5 M_{\odot}$, obtained by comparing the spectroscopic parameters to theoretical models by Martins et al. (2005). From this, the authors determine a full spectroscopic orbital solution via disentangling, enforc- ing a mass ratio of $q=0.175$ as a starting point. They obtained $T_{\text {eff, } 1}=33300 \pm 400 \mathrm{~K}$ and $T_{\text {eff, } 2}=15800 \pm 700 \mathrm{~K}$. Furthermore, Mayer et al. (2013) claimed that while the derived primary radius is typical for an O8V star, the solution for the secondary revealed a smaller radius than predicted for its mass. From their solution, Mayer et al. (2013) determined an age of $\tau=3.3 \pm 0.2 \mathrm{Myr}$ via comparison with rotating theoretical evolutionary tracks (Brott et al. 2011).

Using SAM/NACO measurements obtained with the VLTI, Sana et al. (2014) detected a close bright $\left(\Delta H_{\mathrm{mag}}=2.36\right) \mathrm{com}-$ panion to the inner binary at a separation of $\rho=30 \pm 16$ mas, and confirmed the presence of a bright companion $\left(\Delta H_{\mathrm{mag}}=3.36\right)$ at a separation of $\rho=1.93 \pm 0.04$ arcsec that was originally detected by Mason et al. (1998). Additionally, Sana et al. (2014) detected two fainter companions $\left(\Delta H_{\mathrm{mag}}>5\right)$ at separations larger than 6.5 arcsec. Including the two distant fainter companions, HD 165246 is a sextuple system with the $4.6-\mathrm{d} \mathrm{O}+\mathrm{B}$ eclipsing binary. Without further interferometric observations, additional components interior to the companion at 30 mas cannot be ruled out.

After the failure of a second reaction wheel in 2013, the Kepler satellite was re-purposed as the $K 2$ mission, which scanned the ecliptic in 90-d long pointings (Howell et al. 2014). Owing to the densely crowded field in $K 2$ Campaign 9, Johnston et al. (2017) extracted a $\sim 30$-d light curve using a custom aperture mask, remarking that the mildly saturated pixels, systematics, and thruster firings rendered the remaining $\sim 60$-d segment of the lightcurve of too poor quality for science. Assuming a circular orbit, and adopting the mass ratio and primary effective temperature reported by Mayer et al. (2013), Johnston et al. (2017) determined an updated binary model. Investigation of the residuals after subtraction of the optimised binary model revealed multi-periodic variability on time scales between several hours and a few days. Additionally, highorder harmonics of the orbital frequency as well as a harmonic series whose base frequency is consistent with the rotational frequency of the primary were observed. However, the orbital harmonics may have originated from the assumed circular orbit, which can be mitigated with a larger spectroscopic data set. The authors also note the possibility of Doppler beaming being present, but relegate investigation to future work.

From their binary model, Johnston et al. (2017) estimated 18 per cent contaminating light in the light curve, which is within the 7 to 43 per cent third light contribution estimated from the magnitude contrasts for the other members of the sextuple system (Sana et al. 2014). This estimate depends on the primary effective temperature and mass ratio adopted from Mayer et al. (2013), and as such is subject to change. Finally, Johnston et al. (2017) concluded that the variability signal likely originates from the primary, but suggest that further follow-up is required for unambiguous characterization.

\section{PHOTOMETRIC ANALYSIS}

The process of optimising the binary and atmospheric solutions is an iterative one, with the binary model relying on information determined from an atmospheric solution and vice versa. Hence, we start with an atmospheric solution, then build a binary model, and repeat the atmospheric modelling with the updated information from the binary model. This process is repeated until both solutions no longer change within the output uncertainties. For clarity, we describe the binary and atmospheric processes separately. 


\subsection{K2 Photometry}

Due to the brightness of HD 165246 , the $K 2$ pixels saturate during the 30-min-cadence mode observations. Additionally, HD 165246 lies in a crowded field towards the galactic centre of the Milky Way and shows potentially contaminating sources in the surrounding pixels. To address this, Johnston et al. (2017) built a custom pixel mask that only included the saturated columns and extracted a custom light curve (hereafter LC-A). Due to thruster firings and the saturated and CCD-bleed columns, however, Johnston et al. (2017) only recovered $\mathrm{a} \sim 29.9 \mathrm{~d}$ light curve that was of high enough quality for scientific analysis.

Johnston et al. (2017) concluded that although they identified significant periodicities consistent with both $p$ - and $g$-mode oscillations, due to the limited time-base of their light curve and lack of an independent means of identifying the origin of the oscillations in combination with the presence of contaminating objects in the pixel image, interpretation of the signal was limited. To increase the frequency resolution of the light curve, we extract a new, longer time-base light curve constructed via the halo photometry method (hereafter LC-в). In this method, the scattered light from bright targets across the entire pixel image are collected, with relative weights given to each pixel to scale their contribution to the resulting light curve (White et al. 2017; Pope et al. 2019). Whereas LC-A only has a time-base $T_{A} \sim 29.9 \mathrm{~d}$, LC-B has a time-base of $T_{B} \sim 71.3 \mathrm{~d}$, improving the formal frequency resolution from $d f_{A}=1 / T_{A}=0.034 \mathrm{~d}^{-1}$ for LC-A to $d f_{B}=1 / T_{B}=0.014 \mathrm{~d}^{-1}$ for LC-B. However, due to the weighting scheme used by the halo photometry method, all additional sources present in the mask contribute contaminating signal to the light curve. This not only increases the overall 'third light', but also potentially adds new variability with an unknown amplitude from a contaminating source. Since we know neither whether these separate sources are in fact variable, nor with what amplitudes they may be varying, it is difficult to determine their individual contributions to LC-B. A comparison of the light curves extracted by Johnston et al. (2017) (grey) and in this work (black) are shown in Fig. 1. The clear difference in eclipse depth is caused by the large increase in contaminating light included in LC-B.

\subsection{Updated binary model}

This section discusses the setup for modelling LC-A. The modelling of LC-B is carried out differently, as discussed below. Our initial binary model is based on the solution of Johnston et al. (2017), but incorporates the updated eccentricity and argument of periastron (see Section 4.1.1), as well as the primary effective temperature (see Section 4.3). We optimise our binary model using a Bayesian Markov Chain Monte Carlo (MCMC) numeric sampling code emcee developed by Foreman-Mackey et al. (2013) and draw uncertainties from the posteriors, as was done by Johnston et al. (2017). We calculate the binary model using the ellc code (Maxted 2016). In a recent head-to-head comparison, ellc, PHOEBE 1 (Prsa et al. 2011), PHOEBE2 (Prša et al. 2016), JKTEBOP (Southworth 2013) and WD2007 (Van Hamme \& Wilson 2007) were all shown to agree to $\sim 0.4 \%$ in the reported fundamental parameters of of the eclipsing binary AI Phe (Maxted et al. 2020). While the authors point out that formal uncertainties are often under-estimated when drawn directly from a co-variance matrix, the consistency of their solutions demonstrate that we should not expect any systematic offsets in our solution compared to that of Johnston et al. (2017) based on the use of a different code. In order to incorporate the spectroscopic information, we impose Gaussian priors on the eccentricity $e \sim \mathcal{N}(0.029,0.003)$, the argument of periastron, $\omega_{0} \sim \mathcal{N}(1.63,0.09) \mathrm{rad}$, the semi-amplitude of the primary $K_{1} \sim \mathcal{N}(53.0,0.2) \mathrm{km} \mathrm{s}^{-1}$, the effective temperature of the primary $T_{\text {eff, } 1} \sim \mathcal{N}(36150,600) \mathrm{K}$, and the projected rotational velocity of the primary $v_{1} \sin i \sim \mathcal{N}(268,25) \mathrm{km} \mathrm{s}^{-1}$. The remaining parameters listed in Table 1 are given uniform priors. We allow the mass ratio, $q=M_{2} / M_{1}=K_{1} / K_{2}$, to vary freely so as to not bias the fitting result. Similarly, we allow the third light, $l_{3}$, to vary freely as well.

Instead of directly fitting for the effective temperatures, ellc fits for the surface brightness ratio. To this end, the effective temperature and surface gravities of each component are only used to interpolate values for the limb-darkening and gravity-darkening coefficients in the Kepler passband from the tables published by Claret $\&$ Bloemen (2011). As such, we sample the effective temperatures of each component and use the surface gravities computed for each model as inputs to interpolate for the limb- and gravity darkening coefficients. Following the suggestion of Johnston et al. (2017), we include Doppler boosting in our model in order to account for the asymmetric out-of-eclipse photometric variability observed in the residuals. We include both the light curve and radial velocity (RV) measurements in our fitting procedure.

We run 10000 iterations with 128 individual chains in our MCMC optimisation routine, discarding those iterations which occurred before five times the auto-correlation time as burn-in. The parameter estimates and uncertainties listed in Table 1 are calculated as the median and 68.3-percentile highest posterior density (HPD) confidence interval estimates of the marginalised posteriors for each parameter. The derived quantities, such as the masses and radii of each component, are calculated at each iteration and saved along with the other sampled parameters, allowing us to calculate the estimates and uncertainties for these parameters in the same way. The best fit model, shown in black in the top panel of Fig. 2, is calculated from the values listed in Table 1 . The residuals shown in the bottom panel of Fig. 2 have a root-mean-square (RMS) scatter of 0.89 mmag. We note that the residuals calculated for the same model but without Doppler boosting have an RMS scatter of 0.91 mmag, and display a brightening event at $\Phi \sim 0.3$, which is consistent with the phase when the O-star is accelerating towards the line-of-sight. Furthermore, we note that a significant peak is present at the orbital frequency in the periodogram of the residuals for the model without beaming included. This peak is not detected in the residuals of the model with beaming included.

Beyond the inclusion of the eccentricity, boosting factor, and updated $T_{\mathrm{eff}, 1}$ in our improved binary model, we find a lower mass ratio compared to that of Mayer et al. (2013). We note that this may be caused by the inclusion of third light in our model. The estimated third light contribution of $26_{-1}^{+2}$ per cent is within the estimates of 7 43 per cent expected from the other members of the sextuple system as derived from the K-band magnitude contrasts published by Sana et al. (2014). As a combined result, we calculate that the primary has $M_{1}=23.7_{-1.4}^{+1.1} \mathrm{M}_{\odot}$ with $R_{1}=7.3_{-0.4}^{+0.3} \mathrm{R}_{\odot}$, and the secondary has $M_{2}=3.8_{-0.5}^{+0.4} \mathrm{M}_{\odot}$ with $R_{2}=2.4_{-0.1}^{+0.3} \mathrm{R}_{\odot}$.

As mentioned previously, the modelling of LC-B is conducted differently to that of LC-A. Since we expect the physical binary model to be the same, but the third light contribution to be different, we fix the model and sample only the third light contribution for LC-B. From this, we find that LC-B has roughly 66 per cent composite contaminating light, which is significantly larger than the range expected from the other members of the sextuple system. This sug- 


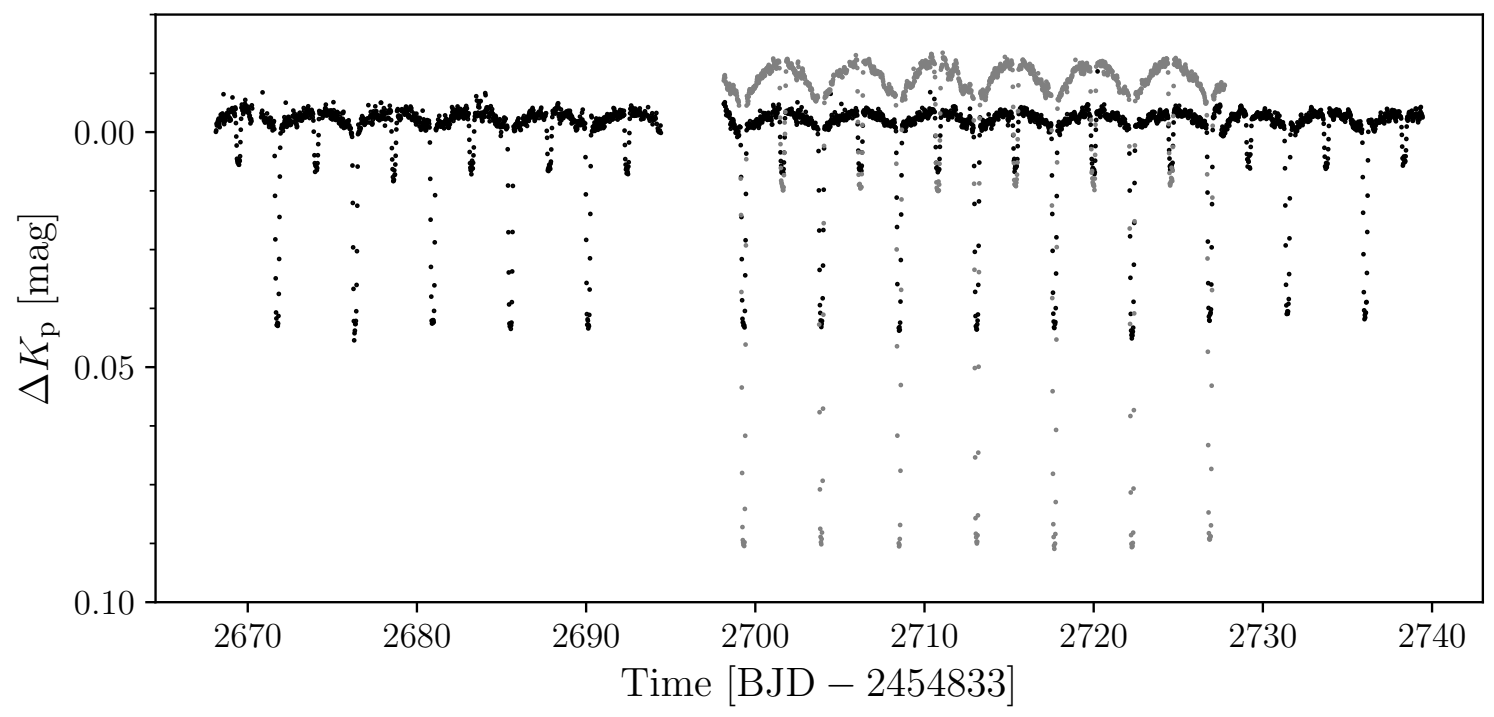

Figure 1. Custom extracted $K 2$ light curve of HD 165246 from Johnston et al. (2017) (LC-A; grey) and halo photometry light curve of HD 165246 (LC-B; black).

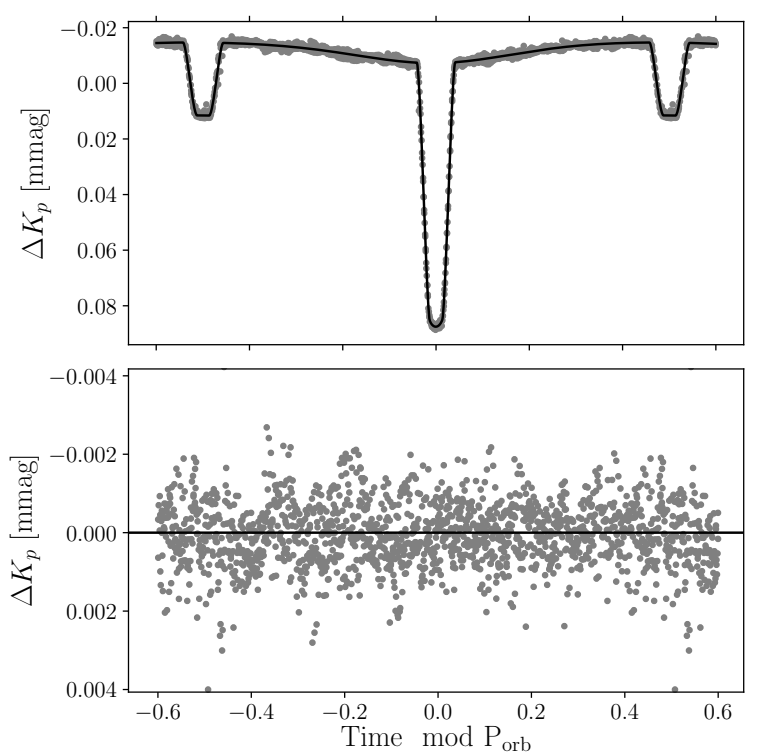

Figure 2. Top: $K 2$ observations (grey) and best fit model (black) for HD 165246. Bottom: Residuals (grey) after removal of best fit model.

gests that this light contains rescaled contributions from other stars in the $K 2$ pixel image.

\subsection{Photometric variability}

We use the residuals of LC-A and LC-B after removal of the optimised binary model (hereafter RES-A and RES-B, respectively) to calculate a Lomb-Scargle periodogram with the aim of searching for significant periodicities. The periodograms for RES-A and RES-B are shown in the top and bottom panels of Fig. 3. We subject both RES-A and RES-B to an iterative prewhitening process to extract all variability with an amplitude signal-to-noise ratio (SNR) greater than four (i.e. SNR $>4$; Breger et al. 1993), where the SNR is calculated over the full range $v \in[0,24.5] \mathrm{d}^{-1}$ up to the Nyquist frequency. Except for one frequency, $f_{14}$, there is no overlap in the extracted frequency lists for RES-A and RES-B.

Given the greater than factor two increase in contaminating light between the two light curves, and lack of similarity between the extracted frequency lists from RES-A and RES-B, we only consider those frequencies extracted from RES-A in our subsequent analyses. The lack of overlap in extracted frequencies between the two lists does not imply that the signal is not present in RES-B. Rather, given the increase in contaminating light, the signal is simply no longer significant according to our $\mathrm{SNR}>4$ criterion. We suggest that the frequencies extracted from RES-B likely originate in one, or several, of the contaminating stars included in the halo-photometry mask, and not from the components of the HD 165246 system. The frequencies extracted from Res-A are given in Table 2. The tabulated frequencies have been filtered for close frequencies occurring within 1.5 times the Rayleigh criterion (Degroote et al. 2009, 2010; Pápics et al. 2012; Bowman 2017).

In contrast to Johnston et al. (2017), we identify only one harmonic of the orbital frequency in RES-A $\left(f_{\mathrm{p}, 5}\right)$. This is a consequence of the improved binary model. As indicated in Table $2, f_{\mathrm{p}, 1}, f_{\mathrm{p}, 2}$, $f_{\mathrm{p}, 3}$, and $f_{\mathrm{p}, 7}$ are also extracted in the LPV analysis in Section 4.1.2. Furthermore, we find nine components $(1,2,3,4,5,6,8,10,11)$ of a harmonic series with $f_{\mathrm{p}, 2}=0.690 \pm 0.003 \mathrm{~d}^{-1}$ as the base frequency. Extended harmonic series are the result of non-sinusoidal signals in the light curve, such as binarity, rotational modulation, or high-amplitude pulsation. The latter option is excluded as all detected amplitudes are below $1 \mathrm{mmag}$. To investigate the former option, we phase RES-A over $f_{\mathrm{p}, 2}=0.69$ in Fig. 4 and find no obvious indication of a blended binary signal. Assuming $f_{\mathrm{p}, 2}$ is the rotation frequency, one expects $F_{1}=f_{\mathrm{p}, 2} / f_{\text {orb }}=3.17 \pm 0.01$, which is within $1-\sigma$ of the value for $F_{1}$ obtained from our binary modelling in Section 3.2, indicating that the rotational interpretation is feasible. Using $f_{\mathrm{p}, 2}=f_{\text {rot }}$ as well as $R_{1}$ and $i$ from Table 1 to compute $v_{1} \sin i$ yields $v_{1} \sin i=253_{-14}^{+11} \mathrm{~km} \mathrm{~s}^{-1}$. This matches well with the value estimated from spectroscopy in Section 4.3. This harmonic rotational signal could be caused by wind variability modulated by the stars rotation (i.e., a clumpy wind), although we do not detect strong wind signatures in the typical diagnostic lines for HD 165246. Nevertheless, the recovery of a significant 
Table 1. Estimated parameters returned from MCMC optimised ellc binary model. We refer to Maxted (2016) for the meaning of the symbols. Quantities marked with * were only used to calculate limb- and gravity-darkening coefficients.

\begin{tabular}{lcc}
\hline Parameter & Unit & Estimate \\
\hline$R_{1} / a$ & - & $0.208_{-0.001}^{+0.0001}$ \\
$R_{2} / a$ & - & $0.069_{-0.002}^{+0.000}$ \\
$a$ & $R_{\odot}$ & $35.3_{-0.7}^{+0.6}$ \\
$S_{b}$ & - & $0.217_{-0.006}^{+0.004}$ \\
$q$ & - & $0.16_{-0.02}^{+0.02}$ \\
$i$ & $\mathrm{deg}$ & $84.0_{-0.1}^{+0.1}$ \\
$P_{\text {orb }}$ & $\mathrm{d}$ & $4.59270_{-0.00001}^{+0.00001}$ \\
$t_{0}$ & $\mathrm{~d}$ & $2457215.4108_{-0.0004}^{+0.0000}$ \\
$f_{c}$ & - & $0.0032_{-0.0008}^{+0.0008}$ \\
$f_{s}$ & - & $0.1656_{-0.0007}^{+0.009}$ \\
$F_{1}$ & - & $3.1_{-0.1}^{+0.2}$ \\
$A_{1}$ & - & $0.30_{-0.2}^{+0.1}$ \\
$B_{1}$ & - & $2.0_{-0.5}^{+0.5}$ \\
$l_{3, a}$ & $\%$ & $26_{-1}^{+2}$ \\
$l_{3, b}$ & $\%$ & $67_{-1}^{+1}$ \\
$T_{\text {eff, } 1}^{*}$ & $\mathrm{~K}$ & $36100_{-200}^{+200}$ \\
$T_{\text {eff }, 2}^{*}$ & $\mathrm{~K}$ & $12100_{-600}^{+600}$ \\
\hline
\end{tabular}

\begin{tabular}{lcc}
\hline \multicolumn{3}{c}{ Derived Parameters } \\
\hline$e$ & - & $0.027_{-0.002}^{+0.003}$ \\
$\omega_{0}$ & $\mathrm{rad}$ & $1.55_{-0.01}^{+0.01}$ \\
$k$ & - & $0.333_{-0.009}^{+0.007}$ \\
$\left(r_{1}+r_{2}\right) / a$ & - & $0.2766_{-0.001}^{+0.002}$ \\
$L_{2} / L_{1}$ & - & $0.0241_{-0.001}^{+0.002}$ \\
$M_{1}$ & $M_{\odot}$ & $23.7_{-1.4}^{+1.1}$ \\
$R_{1}$ & $R_{\odot}$ & $7.3_{-0.4}^{+0.3}$ \\
$\log g_{1}$ & $\mathrm{dex}$ & $4.08_{-0.04}^{+0.02}$ \\
$M_{2}$ & $M_{\odot}$ & $3.8_{-0.5}^{+0.4}$ \\
$R_{2}$ & $R_{\odot}$ & $2.4_{-0.1}^{+0.3}$ \\
$\log g_{2}$ & $\mathrm{dex}$ & $4.26_{-0.14}^{+0.06}$ \\
\hline
\end{tabular}

(SNR $>4)$ signal at $f_{\mathrm{s}, 15} \simeq f_{\mathrm{p}, 2}$ in the LPVs with a full $2 \pi$ variation over the line profile also suggests an interpretation of this signal in terms of rotational modulation.

The Lomb-Scargle periodogram of the prewhitened residuals of both RES-A and RES-B are shown in red in the respective panels of Fig. 3. These periodograms showcase stochastic low-frequency variability as found previously for a large sample of CoRoT, K2, and TESS OB stars (Blomme et al. 2011; Aerts \& Rogers 2015; Aerts et al. 2018; Simón-Díaz et al. 2018; Bowman et al. 2019b,a). Such a signal is predicted independently by 3D hydrodynamic simulations carried out by Edelmann et al. (2019) as well as by different 2D hydrodynamic simulations by Horst et al. (2020) and Ratnasingam et al. (2020). All of these simulations concerned single, young stars. However, given the complexity of this multiple system, other physical causes of this excess may be relevant as well. The stochastic variability occurs in both RES-A and RES-B and is significant according to the $\mathrm{S} / \mathrm{N}>4$ level when the latter is computed from the residual periodogram computed from zero frequency up to the Nyquist frequency (see the dashed-dot horizontal line in Fig. 3). Our argument to consider such a broad range of frequencies to compute the noise level follows Blomme et al. (2011) and Bowman et al. (2020), who

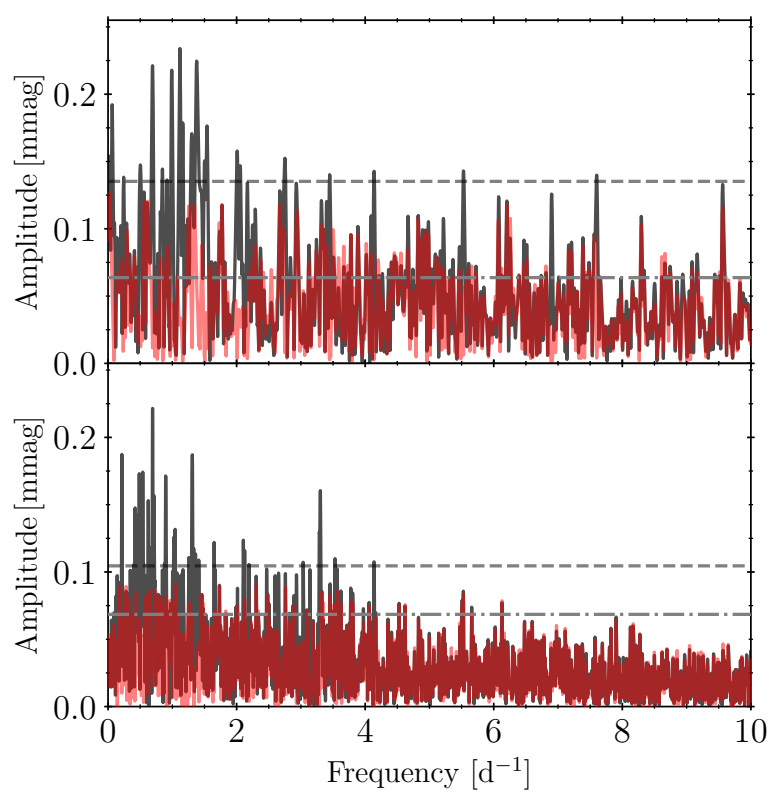

Figure 3. Top: Lomb-Scargle Periodogram of RES-A (black) and its residuals (red). Bottom: Lomb-Scargle Periodogram of RES-B (black) and its residuals (red). Horizontal dashed dark grey and dashed-dotted light grey lines denote four times the white noise level in the residual periodograms after binary model removal and prewhitening, respectively.

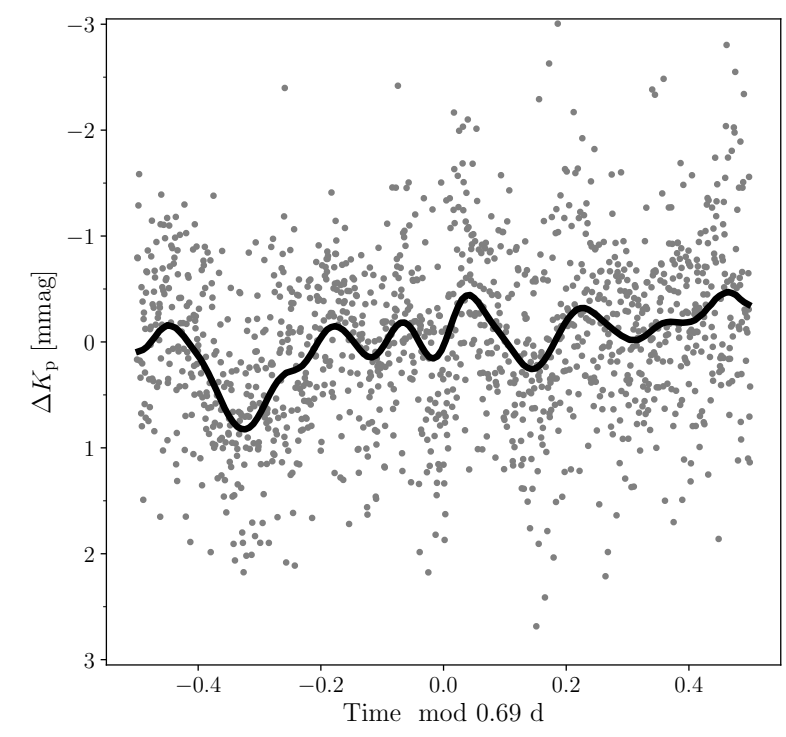

Figure 4. RES-A phase folded over $f_{\mathrm{p}, 3}$. Original data in grey, binned data in black.

have shown that young massive $\mathrm{O}$ stars such as the primary component of HD 165246 have significant low-frequency variability up to frequencies of order $100 \mathrm{~d}^{-1}$.

\section{SPECTROSCOPIC ANALYSIS}

We also investigate the presence of line-profile variability in HD 165246 on various time scales. To do this, we obtained 160 observations between 3 May 2017 and 10 October 2019 with the HERMES spectrograph $(\mathrm{R}=85000)$ (Raskin et al. 2011) attached to 
Table 2. Iterative prewhitening results for the residuals of RES-A.

\begin{tabular}{ccccc}
\hline ID & $\begin{array}{c}\text { Frequency } \\
{\left[\mathrm{d}^{-1}\right]}\end{array}$ & $\begin{array}{c}\text { LC-A } \\
\text { Amplitude } \\
{[\text { [mmag] }}\end{array}$ & SNR & Note \\
\hline$f_{\mathrm{p}, 1}$ & $0.502 \pm 0.004$ & 0.15 & 4.61 & $f_{\mathrm{s}, 9}$ \\
$f_{\mathrm{p}, 2}$ & $0.690 \pm 0.003$ & 0.22 & 6.26 & $f_{\mathrm{s}, 15}$ \\
$f_{\mathrm{p}, 3}$ & $0.992 \pm 0.003$ & 0.23 & 6.45 & $f_{\mathrm{s}, 17}$ \\
$f_{\mathrm{p}, 4}$ & $1.117 \pm 0.003$ & 0.23 & 6.55 & - \\
$f_{\mathrm{p}, 5}$ & $1.305 \pm 0.004$ & 0.15 & 4.59 & $6 f_{\text {orb }}$ \\
$f_{\mathrm{p}, 6}$ & $1.382 \pm 0.003$ & 0.21 & 6.20 & $2 f_{\mathrm{p}, 3}$ \\
$f_{\mathrm{p}, 7}$ & $1.502 \pm 0.004$ & 0.14 & 4.59 & $f_{\mathrm{s}, 14}$ \\
$f_{\mathrm{p}, 8}$ & $1.540 \pm 0.003$ & 0.18 & 5.22 & - \\
$f_{\mathrm{p}, 9}$ & $2.004 \pm 0.003$ & 0.16 & 4.92 & - \\
$f_{\mathrm{p}, 10}$ & $2.057 \pm 0.004$ & 0.15 & 4.68 & $3 f_{\mathrm{p}, 3}$ \\
$f_{\mathrm{p}, 11}$ & $2.165 \pm 0.004$ & 0.14 & 4.45 & - \\
$f_{\mathrm{p}, 12}$ & $2.755 \pm 0.004$ & 0.16 & 4.70 & $4 f_{\mathrm{p}, 3}$ \\
$f_{\mathrm{p}, 13}$ & $3.448 \pm 0.004$ & 0.13 & 4.28 & $5 f_{\mathrm{p}, 3}$ \\
$f_{\mathrm{p}, 14}$ & $4.139 \pm 0.004$ & 0.14 & 4.68 & $6 f_{\mathrm{p}, 3}$ \\
$f_{\mathrm{p}, 15}$ & $5.529 \pm 0.004$ & 0.15 & 4.64 & $8 f_{3}$ \\
$f_{\mathrm{p}, 16}$ & $6.900 \pm 0.004$ & 0.15 & 4.16 & $10 f_{\mathrm{p}, 3}$ \\
$f_{\mathrm{p}, 17}$ & $7.601 \pm 0.004$ & 0.13 & 4.55 & $11 f_{\mathrm{p}, 3}$ \\
\hline & & & &
\end{tabular}

the 1.2-m Mercator telescope at El Observatario Roque de los Muchachos in Santa Cruz de La Palma. As many as 20 consecutive exposures were taken during eight nights in order to achieve a high temporal resolution. The observations have a mean $\mathrm{SNR}=85$ at $550 \mathrm{~nm}$ (ranging from SNR=63 for the lowest quality observation to $\mathrm{SNR}=112$ for the highest quality observation), with an average integration time of $1200 \mathrm{~s}$, and are well distributed across the orbit. These observations were subjected to background and bias subtraction, flat fielding, wavelength calibration (ThAr lamp spectrum), and order merging using the local HERMEs pipeline. The reduced spectra were subsequently normalised via spline fitting.

To maximise the SNR for the spectra, we calculate a Least Squares Deconvolved (LSD) profile (Donati et al. 1997; Tkachenko et al. 2013). This method involves convolving a series of $\delta$ functions of given depths at a given set of wavelengths corresponding to a pre-determined mask to produce an average line profile from the entire spectrum. Thus, the expected increase in SNR is proportional to $\sqrt{N}$, where $N$ is the number of spectral lines used in the mask. Furthermore, by allowing for the simultaneous calculation of multiple average profiles, the LSD methodology enables the detection of multiple components in the spectrum. Whereas the spectra of $\mathrm{O}$-stars feature strong HE II lines, the optical spectra of B-type or cooler stars feature strong HE I or metal lines, depending on the effective temperature. To this end, the use of different masks allows for the detection of multiple components should they have a significant light contribution.

Following these considerations, we subject all of the available HERMES spectra to this method considering two different masks: i) helium lines between 4900 and 5900Å, and ii) metal lines, both of which were constructed from the VALD database (Kupka et al. 1999). Since hydrogen and helium lines are known to suffer from Stark broadening and the signature of radiation driven winds (should they be present), these lines are generally avoided in line profile variability studies. However, in the case of hot rapidly rotating stars, helium lines may be the only lines with sufficient SNR to be considered reliable (Balona et al. 1999; Rivinius et al. 2003). Figure 5 shows the average LSD profiles constructed using the helium-line mask (black) and the metal-line mask (grey). The average metal-line profile exhibits a trend in the red wing. Although

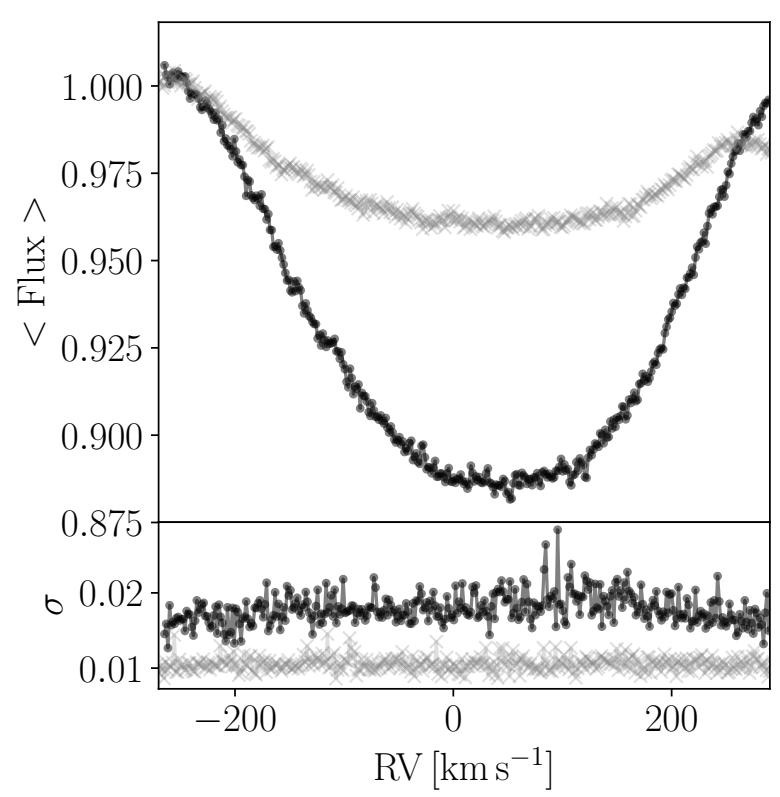

Figure 5. Top: Mean LSD profile for mask containing only He lines between 4900 and $5900 \AA$ (black) and only metal lines across the whole wavelength range (grey). Bottom: Standard deviation across LSD profiles.

it is unclear what introduces this ubiquitous trend in the LSD profiles produced with the metal-line mask, it is clear that these profiles are unsuitable for line profile analysis. The average helium-line profile is well behaved. As such, the remainder of the orbital and line profile analysis is carried out using the LSD profiles produced with the helium-line mask.

\subsection{Line profile variability}

The overall position and shape of a line profile is the combination of extrinsic and intrinsic broadening effects and perturbations, such as RV shifts due to binarity, broadening due to rotation, micro- or macroturbulence, and stellar pulsations, some of which are variable in time. Thus, studying the line profile variations (LPVs) over time allows for the investigation of these signals.

The velocity field produced by coherent/stochastic stellar pulsations induces strictly-/quasi-periodic variations in the lineforming regions near the stellar surface. These variations are detectable via time-resolved spectroscopic observations. Whereas pressure waves have a predominantly radial contribution to the line profile (Aerts \& De Cat 2003), gravity waves produce predominantly tangential velocity variations in the line profile (De Cat \& Aerts 2002). It is worth noting that, although they are expected to be weak in late $\mathrm{O}$ dwarfs, line-driven winds of massive stars are thought to be inherently unstable, introducing yet an additional cause of variability into the line forming region (Puls et al. 2008; Sundqvist et al. 2011). However, the observational consequences of such winds are only important in cases where they are evident in the observations. Moreover, wind variability is stochastic and readily distinguishable from strictly-periodic coherent oscillation modes.

Spectroscopic frequency analysis of coherent pulsation modes employs one of two methods: i) the moment method (Balona 1986; Aerts et al. 1992; Briquet \& Aerts 2003), or ii) the pixel-by-pixel method (Schrijvers et al. 1997; Mantegazza et al. 2000; Zima 2006; Zima et al. 2006). The moment method involves numerically integrating the moments of the extracted spectral line to describe 
the variability in terms of the equivalent width (0th moment), the centroid velocity (corresponding to RV; 1 st moment), profile width (2nd moment), and profile skewness (3rd moment). The moment method is most robust for cases where the star is not rapidly rotating $\left(v \sin i<50 \mathrm{~km} \mathrm{~s}^{-1}\right)$. There are some notable exceptions, such as for studying rotational variability in rapidly rotating chemically peculiar stars (e.g. Lehmann et al. 2006). However, it can still be useful for identifying periodicities when combined with the pixelby-pixel method for analysis of rapidly-rotating stars. In contrast to the moment method that relies on the statistical properties of a line profile, the pixel-by-pixel method relies on the phase and amplitude caused by a stellar pulsation mode across the line profile. While the pixel-by-pixel method is more useful in cases where $v \sin i \gtrsim 50 \mathrm{~km} \mathrm{~s}^{-1}$, it is limited by SNR and is not coupled to the theory of non-radial oscillations as is the case with the moment method. We use the FAMIAS software package (Zima 2008) to carry out the LPV analysis, using both the moment and pixel-bypixel methods.

\subsubsection{Orbital variability}

The dominant source of variability among the spectra is the RV shift induced by the binary motion. In order to investigate any signal caused by stellar pulsations, we must first effectively model and remove this orbital signal. To do this, we calculate the first moment for all LSD profiles based on the He mask and fit a model to these RV shifts using MCMC, as was done with the eclipse modelling in Section 3.2. We fix the orbital period to $P_{\text {orb }}=4.59270 \mathrm{~d}$ and sample the time of periastron passage $t_{\mathrm{pp}}$, the eccentricity $e$, the argument of periastron $\omega_{0}$, the semi-amplitude of the primary $K_{1}$, and the systemic velocity $\gamma$. We derive estimates and $1 \sigma$ uncertainties as the median and 68.3 percentile HPD of the marginalised posteriors, which are listed in Table 3 . The resulting best fit constructed from these values and the residuals are shown in the top and bottom panels of Fig. 6. The residuals show a peak-to-peak scatter of $\sim 20$ $\mathrm{km} \mathrm{s}^{-1}$, indicating the presence of intrinsic variability. Our values for $K_{1}, \gamma, \omega_{0}$ and $e$ are different to those obtained by Mayer et al. (2013). However, given such a small eccentricity and an argument of periastron near $90 \mathrm{deg}$, differing solutions for small data sets, such as that used by Mayer et al. (2013), are not unexpected.

We do not detect the presence of the secondary in any of the individual spectra, their LSD profiles, or in the first moment of these profiles. Additionally, we subject the original data set to spectroscopic disentangling using FDBinary (Ilijic et al. 2004; Pavlovski \& Hensberge 2005), with a fixed orbital solution according to those values listed in Table 3 and only allow $K_{2}$ to vary, but are not able to reliably determine a solution.

\subsubsection{Intrinsic variability}

We remove the orbital motion of the primary from each of the normalised spectra according to the optimised parameters in Table 3. Following this, we calculate new LSD profiles using the helium line mask. These LSD profiles are then used in an LPV analysis, where each LSD profile is assigned a weight according to its SNR. We calculate the zeroth, first, second, and third moments for the data set and subject them to iterative prewhitening until all periodicities with $\mathrm{SNR}>4$ are removed from the time-series of these four moments. We identify 17 significant frequencies in the different moments and in the variability across the LSD profiles as found by the so-called velocity pixel-by-pixel method (see Zima 2008).

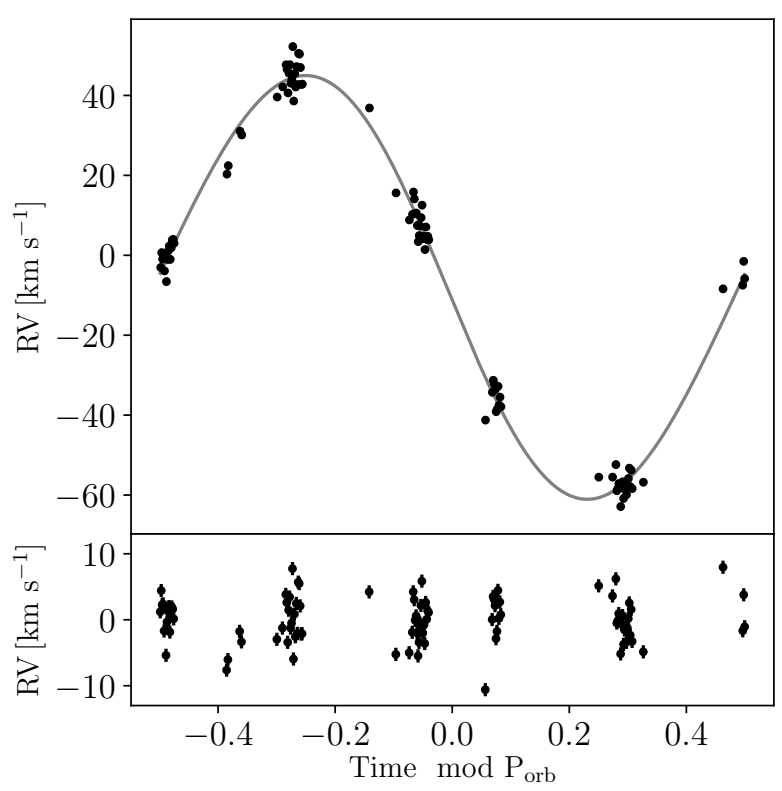

Figure 6. Top: Observed RVs for HD 165246A in black, best fit orbital solution in grey. Bottom: Residuals after subtraction of best fit solution. The observed scatter is astrophysical in nature.

\begin{tabular}{lcc}
\hline Parameter & Unit & HPD Estimate \\
\hline$P_{\text {orb }}$ & BJD & 4.59270 (fixed) \\
$t_{\mathrm{pp}}$ & BJD & $2457215.48_{-0.06}^{+0.06}$ \\
$e$ & - & $0.030_{-0.003}^{+0.003}$ \\
$\omega_{0}$ & $\mathrm{rad}$ & $1.63_{-0.09}^{+0.08}$ \\
$K_{1}$ & $\mathrm{~km} \mathrm{~s}^{-1}$ & $53.0_{-0.2}^{+0.2}$ \\
$\gamma$ & $\mathrm{km} \mathrm{s}^{-1}$ & $-7.9_{-0.1}^{+0.1}$ \\
\hline$a_{1} \sin i$ & $R_{\odot}$ & $4.81_{-0.01}^{+0.01}$ \\
$\mathrm{f}(\mathrm{M})$ & $M_{\odot}$ & $0.071_{-0.001}^{+0.001}$ \\
\hline
\end{tabular}

Table 3. Top: HPD estimates and uncertainties for orbital solution. Bottom: Derived values projected distance of the primary to the common center of mass and the binary mass function, and their uncertainties.

In this method one searches for variability that occur across the entire LSD profile in velocity space, at a given frequency. In application, we fix the frequency values as found in the photometry or moments and only retain those frequencies for which variability with a significant $(\mathrm{SNR}>4)$ is detected across the LSD profile. We fix the frequencies in order to obtain the highest-precision fit results for the amplitude and phase behaviour across the LSD profile, as is common practise in such applications (Zima et al. 2006). We show the periodograms of the moments in Appendix A and the amplitude and phase distributions across the LSDs in Fig. 7. We note that 11 of the frequencies recovered from the frequency analysis of the different moments are newly discovered frequencies, while one is also identified in the photometry, i.e. $f_{s, 9}=f_{p, 1}$. The remaining five frequencies recovered via the pixel-by-pixel method are, as expected, also present in either the photometry or spectroscopy.

This LPV frequency analysis result is indicative of low- to highorder p- and g-mode pulsational variability. Indeed, the frequencies found previously in rapidly rotating $\beta$ Cep pulsators are markedly higher than those we find for HD 165246 (e.g. Schrijvers et al. 2004; 
Uytterhoeven et al. 2004, 2005), except for the frequencies $f_{\mathrm{s}, 4}$ and $f_{\mathrm{s}, 10}$. Aside from these two frequencies, all the other frequencies are lower than those of the $\mathrm{p}$ modes found in the CoRoT space photometry of the slowly rotating O-type dwarf HD 46202 (Briquet et al. 2011), which is to date the $\beta$ Cep star with the highest mass $\left(24 \mathrm{M}_{\odot}\right)$ determined from asteroseismic modelling.

As expected we find common frequencies among the various moments deduced from the LSD time series. Matches occur between $f_{\mathrm{s}, 2}$ and $f_{\mathrm{s}, 11}, f_{\mathrm{s}, 6}$ and $f_{\mathrm{s}, 13}$, and $f_{\mathrm{s}, 8}$ and $f_{\mathrm{s}, 16}$. Additionally, we note that $f_{\mathrm{s}, 9}=f_{\mathrm{p}, 1}$. Of the five frequencies recovered by the pixelby-pixel method, three are frequencies from the space photometry and two are from the spectroscopic moments. Of these, we note that $f_{\mathrm{s}, 15}=f_{\mathrm{p}, 2}$ is identified as the rotational frequency. Figure 7 shows the results of optimising the amplitude (top row) and phase (bottom row) and their errors, smoothed over a $15 \mathrm{~km} \mathrm{~s}^{-1}$ window in blue and orange, respectively. Additionally, we plot the average LSD profile in grey. With the exception of $f_{\mathrm{s}, 13}$, the amplitudes across the line profiles are constant within the uncertainties and do not allow us to interpret the results in terms of mode identification. Phase variability as expected for low-amplitude coherent modes can be seen in some of the bottom panels of Fig. 7, particularly for $f_{\mathrm{s}, 13}$. IGWs would result in more chaotic phase variability across the LSD profiles. The phase variability we detect for $f_{\mathrm{s}, 14}, f_{\mathrm{s}, 16}$, and $f_{\mathrm{s}, 17}$ has a similar level to that found for some of the lowest-amplitude high-degree $\mathrm{p}$ modes found in $v$ Cen (Schrijvers et al. 2004), $\lambda$ Sco (Uytterhoeven et al. 2004), and $\kappa$ Sco (Uytterhoeven et al. 2005). In Section 3.3, we argued that $f_{\mathrm{p}, 2}=f_{\mathrm{s}, 15}$ can be explained as the rotation frequency of the primary. The full $2 \pi$ phase variation across the line profile is consistent with this interpretation.

In conclusion, the complex interplay of frequencies, some of which found in both space photometry and high-resolution spectroscopy, is not exceptional (e.g., Cotton et al., submitted, treating the high-mass $\beta$ Cep pulsator $\beta \mathrm{Cru}$ ). This, along with the frequency regime found for the $\mathrm{p}$ modes of the $\mathrm{O} 9 \mathrm{~V}$ slowly rotating $\beta$ Cep star HD 46202 (Briquet et al. 2011), makes us interpret the frequencies detected in the LSD and in the space photometry of HD 165246 as due to a mixture of coherent low-order $p$ and $g$ modes, along with IGWs, shifted into the gravito-inertial regime by the star's fast rotation (Aerts et al. 2019).

\subsection{Interpretation as variable macroturbulence}

It is well known that rotational broadening alone is not sufficient to explain the shape of line profiles in massive stars (Gray 2005). A microturbulent velocity component, which represents turbulent pressure on spatial scales smaller than the mean-free path of a photon, is added during the atmosphere calculations, and can thus alter the line strength and estimated effective temperature. Furthermore, a macroturbulent velocity component, $\xi_{\text {macro, }}$ which represents turbulent pressure on spatial scales larger than the mean-free path of a photon, is required to better reproduce the shapes of massive star line profiles (Howarth et al. 1997; Gray 2005; Aerts et al. 2009; Simón-Díaz \& Herrero 2014). The macroturbulent velocity profile can be either anisotropic (with different radial and tangential contributions) or isotropic (with equal radial and tangential contributions). Moreover, while a microturbulent component is considered during the atmospheric calculations, both macroturbulence and rotational broadening are included via convolution to an already computed spectrum, making their contribution to line shape degenerate. Additionally, those stars which undergo other phenomena such as sub-surface convection, spots, and/or stellar pulsations which impact the shape of the line profile and make it asymmetric
Table 4. Significant frequencies, amplitudes, and SNRs extracted from 0th, 1st, 2nd, and 3rd moments and from the pixel-by-pixel method.

\begin{tabular}{|c|c|c|c|c|}
\hline & $d^{-1}$ & - & SNR & Note \\
\hline \multicolumn{2}{|c|}{ Zeroeth Moment } & $\mathrm{km} \mathrm{s}^{-1}$ & & \\
\hline$f_{\mathrm{s}, 1}$ & $0.18 \pm 0.02$ & $2.3 \pm 0.3$ & 21.6 & - \\
\hline$f_{\mathrm{s}, 2}$ & $0.487 \pm 0.004$ & $0.8 \pm 0.1$ & 8.0 & - \\
\hline$f_{\mathrm{s}, 3}$ & $1.729 \pm 0.003$ & $0.9 \pm 0.2$ & 8.0 & - \\
\hline$f_{\mathrm{s}, 4}$ & $7.342 \pm 0.002$ & $0.8 \pm 0.2$ & 4.1 & - \\
\hline \multicolumn{2}{|c|}{ First Moment } & $\mathrm{km} \mathrm{s}^{-1}$ & & \\
\hline$f_{\mathrm{s}, 5}$ & $1.476 \pm 0.002$ & $2.9 \pm 0.4$ & 7.5 & - \\
\hline$f_{\mathrm{s}, 6}$ & $1.821 \pm 0.002$ & $3.3 \pm 0.2$ & 8.2 & - \\
\hline \multicolumn{2}{|c|}{ Second Moment } & $\mathrm{km}^{2} \mathrm{~s}^{-2}$ & & \\
\hline$f_{\mathrm{s}, 7}$ & $2.225 \pm 0.002$ & $500 \pm 40$ & 12.6 & - \\
\hline$f_{\mathrm{s}, 8}$ & $2.638 \pm 0.003$ & $300 \pm 40$ & 7.2 & - \\
\hline$f_{\mathrm{s}, 9}$ & $0.501 \pm 0.003$ & $400 \pm 100$ & 11.5 & $f_{\mathrm{p}, 1}$ \\
\hline$f_{\mathrm{s}, 10}$ & $4.873 \pm 0.006$ & $240 \pm 40$ & 4.5 & - \\
\hline \multicolumn{2}{|c|}{ Third Moment } & $\mathrm{km}^{3} \mathrm{~s}^{-3}$ & & \\
\hline$f_{\mathrm{s}, 11}$ & $0.489 \pm 0.002$ & $168000 \pm 31000$ & 9.6 & - \\
\hline$f_{\mathrm{s}, 12}$ & $0.800 \pm 0.006$ & $150000 \pm 14000$ & 8.2 & - \\
\hline \multicolumn{2}{|c|}{ Pixel-by-pixel method } & Continuum Units & & \\
\hline$f_{\mathrm{s}, 13}$ & 1.821 (fixed) & $3.1 \pm 0.9$ & 12.4 & $f_{\mathrm{s}, 6}$ \\
\hline$f_{\mathrm{s}, 14}$ & 1.502 (fixed) & $2.9 \pm 1.0$ & 6.9 & $f_{\mathrm{p}, 7}$ \\
\hline$f_{\mathrm{s}, 15}$ & 0.690 (fixed) & $2.7 \pm 1.0$ & 8.2 & $f_{\mathrm{p}, 2}$ \\
\hline$f_{\mathrm{s}, 16}$ & 2.638 (fixed) & $2.1 \pm 1.0$ & 4.8 .8 & $f_{\mathrm{s}, 8}$ \\
\hline$f_{\mathrm{s}, 17}$ & 0.992 (fixed) & $3.9 \pm 2.0$ & 15.3 & $f_{\mathrm{p}, 3}$ \\
\hline
\end{tabular}

require further consideration to accurately reproduce the line profile (Aerts et al. 2014).

A non-radial pulsation produces asymmetric deviations from the static line profile that travel through the line profile over the pulsation phase. Thus, the presence of pulsations can directly influence the measurement of observed quantities, such as $\xi_{\text {macro }}$. Aerts et al. (2009) and Aerts \& Rogers (2015) demonstrated that the collective contribution of stellar oscillation modes and IGWs, respectively, can explain, at least in part, the observed macroturbulence deduced from the spectral-line properties of massive stars. Aerts et al. $(2009,2014)$ also caution that determination of $v \sin i$ can be complicated by the presence of stellar pulsations which induce asymmetric time-dependent variations in the line profile, a problem made worse when spectra obtained at drastically different pulsation phases are stacked. Furthermore, Aerts et al. (2014) show that the macroturbulence needed to explain purely pulsational broadening can be on the order of or larger than the value of the rotational velocity, and is variable over the pulsation cycle.

As we observe pulsations and a large projected rotational velocity in the O-star primary of HD 165246, it is important that we obtain an independent estimate of $v \sin i$ to use as a constraint in the atmospheric modelling. We achieve this by using the IACOB-BROAD tool developed by Simón-Díaz \& Herrero (2014) to independently estimate $v \sin i$ for the cases considering no macroturbulence, using the first moment of the Fourier transform (FT) and performing goodness-of-fit (GOF) calculations, both allowing for isotropic macroturbulence. We select 10 spectra from our data set, five of which span the range of the entire data set and the other five of which were taken on a single night. This allows us to investigate the stability of the $v \sin i$ and $\xi_{\text {macro }}$ estimates over different timescales and at different points along any variability cycle. Given the SNR of our spectra, we use the HE II 4541 line for our calculations. The 


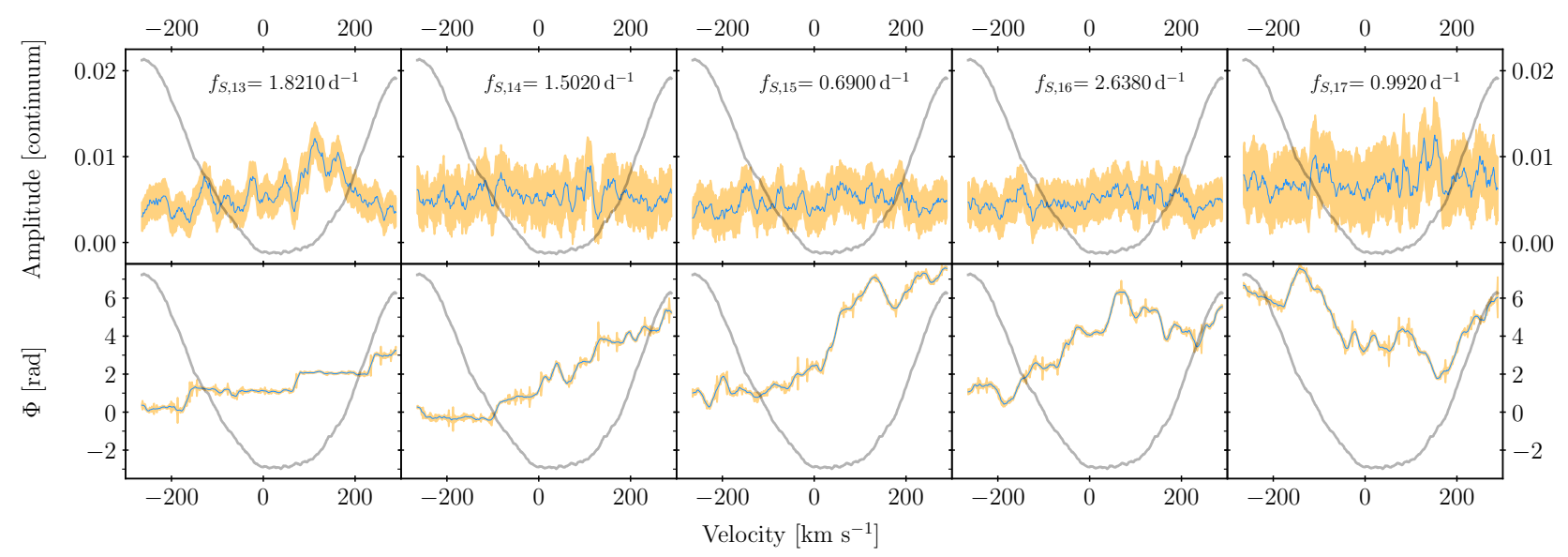

Figure 7. Top: Amplitude across line profile. Bottom: Phase across line profile. Smoothed data displayed in blue, errors displayed in orange. Line profile overplotted in grey in both panels.

results of using IACOB-BROAD on the HE II 4541 line are listed in Table 5.

As expected, the estimates of $v \sin i$ are systematically higher when $\xi_{\text {macro }}$ is fixed at $0 \mathrm{~km} \mathrm{~s}^{-1}$, yielding $v \sin i=268 \pm 25 \mathrm{~km} \mathrm{~s}^{-1}$ compared to $v \sin i_{\mathrm{FT}}=238 \pm 40 \mathrm{~km} \mathrm{~s}^{-1}$ and $v \sin i_{\mathrm{GOF}}=$ $230 \pm 46 \mathrm{~km} \mathrm{~s}^{-1}$. The presence of pulsations in HD 165246, however, complicates the interpretation of this. Aerts et al. (2014) demonstrated that even low amplitude pulsations can produce either over- or under-estimations of $v \sin i$, and hence $\xi_{\text {macro }}$, by the FT method if a simple isotropic model of macroturbulence is used, depending on the pulsation phase of the observed spectrum. This is because a time-independent isotropic velocity is the wrong prior assumption when fitting profiles broadened by time-dependent pulsation modes. From this, Aerts et al. (2014) conclude, in agreement with Aerts et al. (2009), that the best means for estimating $v \sin i$ is via GOF with fixed $\xi_{\text {macro }}=0 \mathrm{~km} \mathrm{~s}^{-1}$. The estimate for $v \sin i\left(\xi_{\text {macro }}=0\right)=268 \pm 25 \mathrm{~km} \mathrm{~s}^{-1}$ is in agreement with the value for $v_{1} \sin i=253_{-14}^{+11} \mathrm{~km} \mathrm{~s}^{-1}$ that is calculated assuming that $f_{\mathrm{p}, 2}$ is the rotation frequency.

Both the GOF and FT methods of determining $\xi_{\text {macro }}$ reveal that the estimates of $\xi_{\text {macro }}$ are variable on both inter- and intranightly timescales, with the mean estimates exceeding $100 \mathrm{~km} \mathrm{~s}^{-1}$. This variability in the estimates of $\xi_{\text {macro }}$ can be understood in terms of the time-dependent asymmetries produced by pulsations in line profiles, which require different amounts of isotropic macroturbulence for a satisfactory fit. Thus, this is not indicative of actual variation in the macroturbulent velocity, but rather the consequence of measuring $\xi_{\text {macro }}$ at different phases of different pulsation cycles. Furthermore, we recall the $18 \mathrm{~km} \mathrm{~s}^{-1}$ peak-to-peak scatter observed in the residuals of the RV fit in Fig. 6. Both the variability in $\xi_{\text {macro }}$ and in the RVs are consistent with the effects of both non-radial coherent $\mathrm{p}$ and g modes, as well as IGWs propagating in the lineforming region of the primary of HD 165246 (Aerts et al. 2014). Such modes and waves occur in the frequency range covered by the values listed in Table 4.

\subsection{Updated atmospheric solution}

To determine an updated atmospheric solution, we co-add the normalised spectra (velocity corrected according to the orbital motion), as shown in blue in Fig. 8. We perform an atmospheric analysis using the numerical setup as described in Abdul-Masih et al. (2019).
In brief, we use a genetic algorithm (GA) wrapped around the nonlocal thermodynamic equilibrium (NLTE) radiative transfer code FASTWIND (Puls et al. 2005) to optimise the atmospheric parameters of the O-star primary (Charbonneau 1995; Mokiem et al. 2005). The GA allows for an efficient sampling of the expansive parameter space and uses a merit function which is proportional to the inverse of the chi-square of a given atmospheric model in comparison to a subset of lines from the co-added observed spectrum.

The parameters for each generation of models are determined by combining parameters from the previous generation, where models with a lower chi-square have a higher chance of passing their parameters to the next generation. At each generation, parameter variations, or mutations, are introduced to effectively sample the parameter space. The GA analysis was carried out iteratively with the binary modelling (discussed in Section 3.2), where the effective temperature was fixed in the binary modelling first, and then the surface gravity and light-dilution from the binary model was fixed in the next iteration of spectral fitting, until convergence was reached.

We perform an 11 parameter optimisation including several stellar parameters: effective temperature $\left(T_{\text {eff }}\right)$, surface gravity $(\log g)$, microturbulent velocity $\left(\xi_{\text {micro }}\right)$ and macroturbulent velocity $\left(\xi_{\text {macro }}\right)$, three wind parameters: the mass loss rate $(\log \dot{M})$, the exponent of the wind velocity profile $(\beta)$ and the terminal wind speed $\left(v_{\text {inf }}\right)$, and four surface abundance parameters: the helium abundance $\left(Y_{\mathrm{He}}\right)$, the carbon abundance $\left(\eta_{\mathrm{C}}\right)$, the nitrogen abundance $\left(\eta_{\mathrm{N}}\right)$ and the oxygen abundance $\left(\eta_{\mathrm{O}}\right)$. The helium abundance is given as the ratio of the helium number density to the hydrogen number density while the abundances of carbon, nitrogen and oxygen are given as the log of the ratio of the elemental number density to the hydrogen number density plus 12 . The final optimised values for the FASTWIND model are listed in Table 6, and the best fit model is shown in red in Fig. 8. Our solution results in a primary effective temperature which is nearly $3000 \mathrm{~K}$ hotter than determined by Mayer et al. (2013). Furthermore, we note that we find a high microturbulent velocity in HD 165246, whereas previous studies typically fix this quantity. Finally, the macroturbulent velocity reported by the GA optimisation is significantly lower than that obtained via the GOF and FT methods previously. This is due to differences in the way that macroturbulence is described, i.e. isotropic in the GOF and FT methods vs. anisotropic in FASTwIND. 
Table 5. Estimates for $v \sin i$ and $\xi_{\text {macro }}$ for 10 spectra.

\begin{tabular}{lccccc}
\hline $\begin{array}{l}\text { BJD } \\
\mathrm{d}\end{array}$ & $\begin{array}{c}v \sin i\left(\xi_{\text {macro }}=0\right) \\
\mathrm{km} \mathrm{s}^{-1}\end{array}$ & $\begin{array}{c}v \sin i_{\mathrm{FT}} \\
\mathrm{km} \mathrm{s}^{-1}\end{array}$ & $\begin{array}{c}v \sin i_{\mathrm{GOF}} \\
\mathrm{km} \mathrm{s}^{-1}\end{array}$ & $\begin{array}{c}\xi_{\text {macro,FT }} \\
\mathrm{km} \mathrm{s}^{-1}\end{array}$ & $\begin{array}{c}\xi_{\text {macro,GOF }} \\
\mathrm{km} \mathrm{s}^{-1}\end{array}$ \\
\hline 2457896.7177510 & 256 & 235 & 234 & 118 & 117 \\
2457962.4721318 & 264 & 239 & 237 & 119 & 118 \\
2457964.5220527 & 282 & 253 & 252 & 136 & 136 \\
2457965.4924402 & 247 & 225 & 232 & 113 & 113 \\
2457965.4999635 & 274 & 235 & 213 & 154 & 189 \\
2457965.5074870 & 265 & 248 & 223 & 120 & 163 \\
2457965.5150105 & 244 & 217 & 207 & 132 & 153 \\
2457965.5225342 & 254 & 226 & 224 & 137 & 137 \\
2457967.4127213 & 333 & 257 & 246 & 222 & 246 \\
2457971.5365183 & 258 & 241 & 237 & 108 & 108 \\
\hline Mean & 268 & 238 & 230 & 136 & 148 \\
Range & 89 & 40 & 46 & 113 & 137 \\
$\sigma$ & 25 & 12 & 13 & 31 & 41 \\
\hline
\end{tabular}

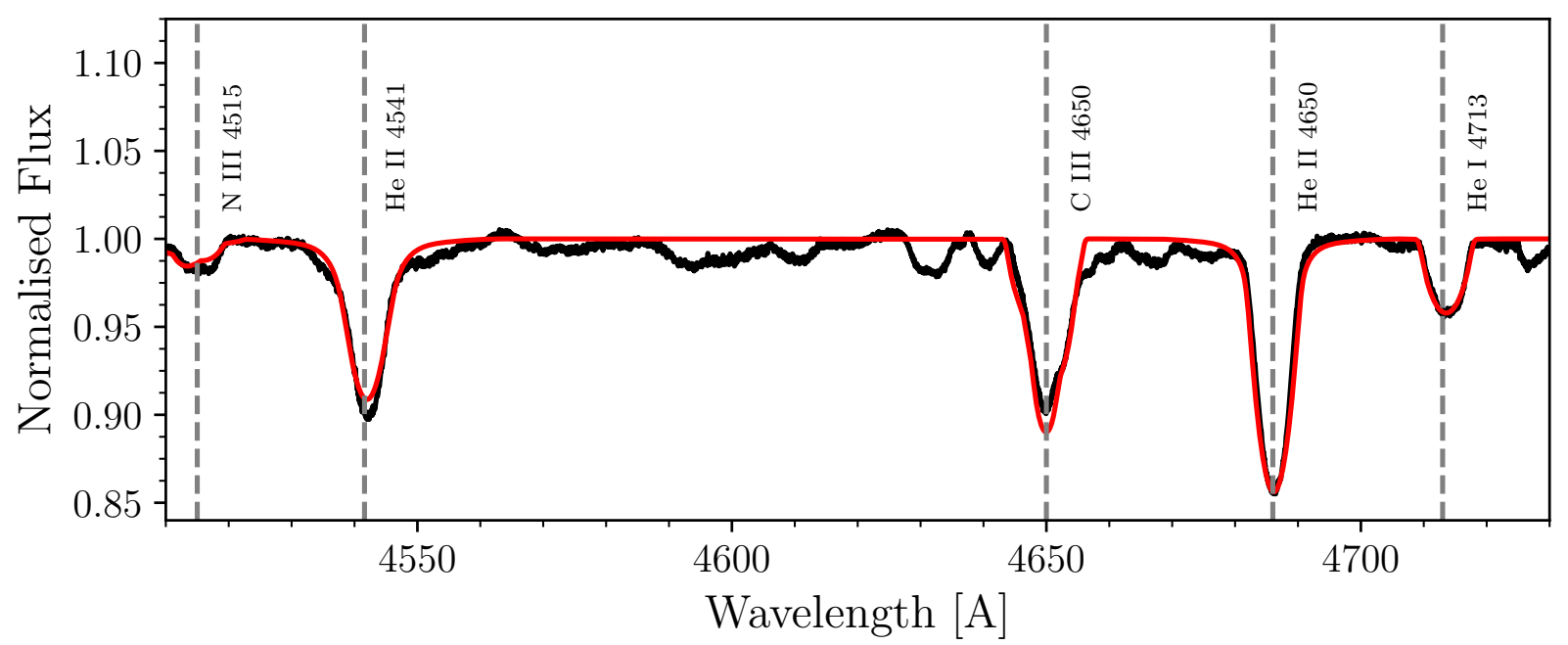

Figure 8. Observed co-added spectrum in black and best fit model according to Table 6 in red. Location and identification for a subset of spectral lines indicated by vertical dashed lines.

\section{DISCUSSION}

We detect multiple sources of variability in both the spectroscopic and photometric observations of HD 165246. We identify a series of harmonics in the $K 2$ photometry whose base frequency, $f_{\mathrm{p}, 2}$, is also present in the HERMES spectroscopy $\left(f_{\mathrm{p}, 15}\right)$. The presence of such harmonics in the photometry indicates the presence of nonsinusoidal signal in the data, which in this context has two potential explanations: i) rotational modulation, or ii) background binary signal. Given the corroboration of the binary modelling, atmospheric modelling, as well as the identification of this signal in both the photometric and spectroscopic time series, we argue that this is the result of rotational modulation on the primary $\mathrm{O} 8 \mathrm{~V}$ star.

Assigning a single underlying mechanism to the remaining variance in the low-frequency regime is challenging since it contains both coherent $\mathrm{p}$ and $\mathrm{g}$ modes self-excited via the $\kappa$-mechanism as well as stochastically excited IGWs, which may also drive modes at resonant eigenfrequencies (Bowman et al. 2019b; Edelmann et al. 2019; Horst et al. 2020). Given the poor frequency resolution of both the spectroscopic and photometric data sets, we are currently unable to identify the modes/waves. Since we do, however, identify signal in both the photometry and spectroscopy independently, we are able to conclusively state that there is significant pulsational variability present at low frequencies which originates from the O-type primary. Moreover, the dominant frequencies detected in the moment variations listed in Table 4 all lead to a ratio of the tangential to radial velocity amplitude above unity. This ratio, otherwise known as a $\mathrm{K}$ value, can be computed from the mass, radius and frequency of the mode following Eq. (3.162) in Aerts et al. (2010). This leads to ratios with a range covering roughly $[3.3,62.2]$, meaning that the pulsational variability is dominated by g modes or IGWs as these have dominant tangential motions, while $\mathrm{p}$ modes are dominated by radial motions. The signal corresponding to $f_{\mathrm{s}, 4}$ and $f_{\mathrm{s}, 10}$ leads to low $\mathrm{K}$ values and may correspond to either low-order p modes (Briquet et al. 2011) or high-order g modes shifted to high frequencies due to the Coriolis force (Buysschaert et al. 2018).

The observed $\xi_{\text {macro }}$ variability may have various candidate sources: i) coherent pulsations, ii) IGWs, iii) sub-surface convection, or iv) stochastic wind variability. As we have identified the presence of pulsations and/or IGWs, these invariably have at least some contribution to the variability in $\xi_{\text {macro }}$ on the basis of their contribution to the line profiles from which $\xi_{\text {macro }}$ is estimated. The parameters of the $\mathrm{O} 8 \mathrm{~V}$ primary place it in a region on the HR 
Table 6. Estimated parameters returned from optimised FASTWIND models.

\begin{tabular}{lcc}
\hline Parameter & Unit & Estimate \\
\hline$T_{\text {eff }}$ & $\mathrm{K}$ & $36200_{-600}^{+900}$ \\
$\log g$ & $\mathrm{dex}$ & $4.05_{-0.15}^{+0.07}$ \\
$\log M$ & $\log \left(M_{\odot} / \mathrm{yr}\right)$ & $-8.0_{-0.2}^{+0.2}$ \\
$\beta$ & - & $0.6787_{-0.65}^{+0.15}$ \\
$v_{\infty}$ & $\mathrm{km} \mathrm{s}^{-1}$ & $2530_{-260}^{+60}$ \\
$\xi_{\text {micro }}$ & $\mathrm{km} \mathrm{s}^{-1}$ & $13_{-1.3}^{+1.0}$ \\
$\xi_{\text {macro }}$ & $\mathrm{km} \mathrm{s}^{-1}$ & $20_{-6}^{+5}$ \\
$\nu$ sin $i$ & $\mathrm{~km} \mathrm{~s}^{-1}$ & $268($ fixed $)$ \\
$Y_{\mathrm{He}}$ & - & $0.092_{-0.004}^{+0.004}$ \\
$\eta_{\mathrm{C}}$ & - & $8.40_{-0.04}^{+0.07}$ \\
$\eta_{\mathrm{N}}$ & - & $7.62_{-0.09}^{+0.1}$ \\
$\eta_{\mathrm{O}}$ & - & $8.67_{-0.01}^{+0.2}$ \\
\hline
\end{tabular}

diagram where the sub-surface convective velocity is theoretically estimated to be below $2.5 \mathrm{~km} \mathrm{~s}^{-1}$ (Cantiello et al. 2009, see their Fig. 9, top panel). Therefore, sub-surface convection cannot fully explain the large and variable tangential velocities that we observe. Furthermore, our estimates of $\xi_{\text {micro }}, \log g$, and $v \sin i$ place the Ostar primary in an underpopulated region of the parameter space to compare with the predictions of Cantiello et al. (2009). This comparison, however, is complicated by the fact that HD 165246 is within the galaxy, whereas the majority of the sample analysed by Cantiello et al. (2009) consists of stars from the Large and Small Magellanic Clouds. In addition, stochastically variable wind signatures as computed by Krtička \& Feldmeier (2018) stem from outflow and hence correspond dominantly to radial motions, while the observations point to dominant tangential velocity variations. Our observations of high $v \sin i$, high $\xi_{\text {macro }}$, and the presence of IGWs in the young O-star primary of HD 165246 are consistent with the results of both Simón-Díaz et al. (2017) and Bowman et al. (2019a, 2020). Simón-Díaz et al. (2017) observe a wide range of $\xi_{\text {macro }}$ from spectroscopy and Bowman et al. (2019a) observe a low-frequency excess in photometry (identified as IGWs) in both galactic and LMC O and B stars across the upper HRD. This suggests a common intrinsic mechanism and a relationship between macroturbulence as found in spectroscopy and stochastic low-frequency variability detected in space photometry (Burssens et al. 2020; Bowman et al. 2020).

As demonstrated in Fig. 9, the O8 V primary is located close to the zero-age main-sequence in reference to both the non-rotating tracks with different amounts on internal chemical mixing (black tracks), calculated according to Johnston et al. (2019a) using MESA (r-10398 Paxton et al. 2018) as well as tracks with an initial rotational velocity of $40 \%$ critical (grey tracks), calculated by Choi et al. (2016) using MESA (r-7503 Paxton et al. 2015). The non-rotating models are calculated such that the internal chemical element mixing is represented in two distinct regimes. The first regime corresponds to the convective boundary mixing (CBM) region, where we represent $\mathrm{CBM}$ with diffusive convective overshooting. For overshooting, the free parameter $f_{\text {ov }}$ scales the slope with which the mixing profile decays beyond the core, as defined by the Schwarzschild criterion in terms of the local pressure scale height, $H_{p}$. These models only account for overshooting extending beyond the convective core, and not around any intermediate convective regions in the envelope. The second regime corresponds to radiative envelope mixing (REM), according to the chemical mixing induced by inter- nal gravity waves as derived by Rogers \& McElwaine (2017) and implemented in MESA models by Pedersen et al. (2018). In this profile, the free parameter $D_{\text {REM }}$ sets the base efficiency of chemical mixing induced by this mechanism, and has units of $\mathrm{cm}^{2} \mathrm{~s}^{-1}$. The dashed black (non-rotating) models plotted in Fig. 9 represent the case of minimal internal chemical mixing, with $f_{\mathrm{ov}}=0.005$ and $D_{\text {REM }}=10 \mathrm{~cm}^{2} \mathrm{~s}^{-1}$. The dashed-dotted black (non-rotating) models represent the case of maximum internal mixing with $f_{\mathrm{ov}}=0.04$ and $D_{\text {REM }}=10000 \mathrm{~cm}^{2} \mathrm{~s}^{-1}$. These limiting values are those deduced by asteroseismology of intermediate-mass g-mode pulsating field stars (Briquet et al. 2007; Daszyńska-Daszkiewicz \& Walczak 2010; Daszyńska-Daszkiewicz et al. 2013; Moravveji et al. 2016; Schmid \& Aerts 2016; Buysschaert et al. 2018; Walczak et al. 2019; Wu \& Li 2019). Despite the differences in the form of internal mixing between the two sets of evolutionary tracks, comparison of the observations with both tracks reveals a massive primary which has consumed less than $30 \%$ of its initial core hydrogen content, as seen in Fig. 9.

Figure 10 compares the dynamical mass and surface gravity estimates for the O8V primary of HD 165246 with theoretical isochrones with different amounts of internal mixing using models calculated using MESA (Paxton et al. 2018) by Johnston et al. (2019a). The location of HD 165246A in Fig. 10 indicates that it has an age between 2-3 Myr and a core hydrogen content of $X_{c}=0.54$, given the uncertainties on the dynamical surface gravity (black), or an age between 2-4.5 Myr given the uncertainties on the spectroscopic surface gravity (red). Our age estimates agree with those of Mayer et al. (2013) who used evolutionary tracks from Brott et al. (2011). The overlap between the dynamical and spectroscopic surface gravity estimates provides support for the mass estimate derived for the primary component. However, given the inability to detect the secondary star in the spectra, we have no means of independently verifying the solution for the secondary, making us wary of its absolute dimensions. With this in mind, we find that the secondary is not yet on the main-sequence when compared to the isochrones in Fig. 10. We note that without smaller uncertainties on the parameters of the primary, we are not able to constrain the impact of internal chemical mixing on the evolution of this star. Additionally, without proper characterization of the secondary via detection of its RV variations, we are not able to perform isochronecloud fitting as introduced by Johnston et al. (2019b) and applied by Tkachenko et al. (2020).

\section{CONCLUSIONS}

This work made use of an extensive set of new time-series spectroscopy to build upon the work by Mayer et al. (2013) and Johnston et al. (2017) for a more in-depth study of the massive eclipsing $\mathrm{O}+\mathrm{B}$ binary HD 165246. Using this spectroscopy, we obtained updated atmospheric and binary solutions revealing an effective temperature of $T_{\text {eff }}=36000 \mathrm{~K}$ and a mass of $M=23.7 \mathrm{M}_{\odot}$ for the O-star primary. Furthermore, we determine a rotation rate of $v \sin i=268 \mathrm{~km} \mathrm{~s}^{-1}$, microturbulence of $\xi_{\text {micro }}=13 \mathrm{~km} \mathrm{~s}^{-1}$, and variable macroturbulence linked to time-dependent pulsational line-broadening. We explored the variability detected in both the $K 2$ photometry and HERMES spectroscopy, and find it consistent with rotational modulation, as well as intrinsically excited stellar pulsations.

The presence of pulsational variability in the light curve and HERMES spectroscopy provide at least a partial explanation for the high macroturbulence derived from the atmospheric modelling. This is consistent with the predictions of Aerts et al. $(2009,2014)$ 


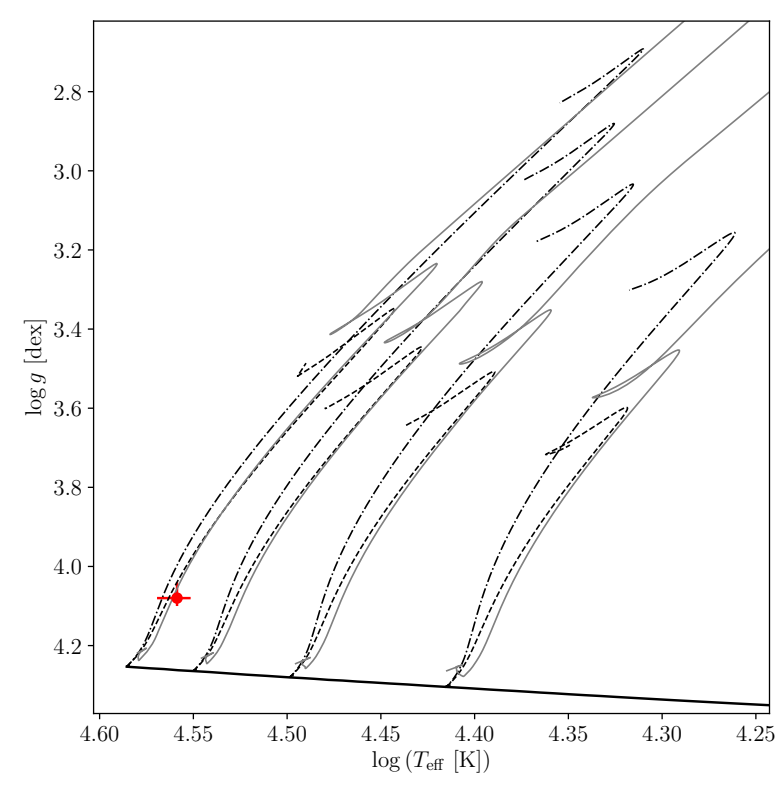

Figure 9. Evolutionary tracks for 25, 20, 15, and $10 \mathrm{M}_{\odot}$ models. Solid black line denotes ZAMS line. Black tracks taken from Johnston et al. (2019a) with $f_{\mathrm{ov}}=0.005, D_{\mathrm{REM}}=10 \mathrm{~cm}^{2} \mathrm{~s}^{-1}$ (dashed lines) and $f_{\mathrm{ov}}=0.040$, $D_{\text {REM }}=10000 \mathrm{~cm}^{2} \mathrm{~s}^{-1}$ (dashed-dotted lines). Black tracks have $\mathrm{Y}=0.276$, $\mathrm{Z}=0.0140$, and use OP opacities. Solid grey tracks are MIST Stellar evolution tracks taken from Choi et al. (2016) with $v_{\mathrm{ZAMS}} / v_{\text {crit }}=0.4, \mathrm{Y}=0.2703$, $\mathrm{Z}=0.0142$, and OPAL opacities, for the same masses.

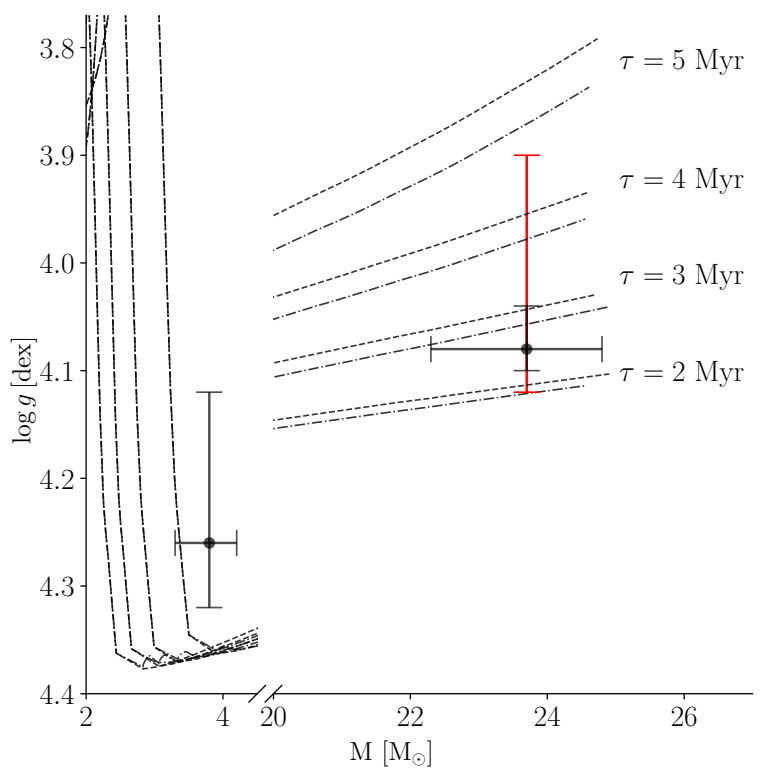

Figure 10. Isochrones for 2,3,4, and $5 \mathrm{Myr}$ shown in grey for tracks with $f_{\mathrm{ov}}=0.005, D_{\mathrm{REM}}=10 \mathrm{~cm}^{2} \mathrm{~s}^{-1}$ (dashed lines) and $f_{\mathrm{ov}}=0.040$, $D_{\text {REM }}=10000 \mathrm{~cm}^{2} \mathrm{~s}^{-1}$ (dashed-dotted lines). All models have $\mathrm{Y}=0.276$ and $\mathrm{Z}=0.014$ and OPAL opacities. Dynamical (spectroscopic) estimates for mass and $\log g$ denoted by black (red) error-bars. and the observations of Simón-Díaz et al. (2017). The evolutionary status of the $\mathrm{O} 8 \mathrm{~V}$ primary is consistent with expectations from models. Higher precision on the fundamental stellar parameters is required for scrutinising evolutionary models at masses and ages typical of O-stars. However, the observed atmospheric properties of the primary component situate this star in an underpopulated region of the parameter space of massive star variability studies.

High-mass eclipsing binaries such as HD 165246 are integral to the study of variability in the upper regions of the HRD. The characterization of such systems is required to provide benchmarks for hydrodynamical simulations and evolutionary codes. Currently, the community lacks the sample size of well characterized high-mass stars (including fundamental parameters) required to discriminate between the physical mechanisms proposed as the causes of observed variability. The Transiting Exoplanet Survey Satellite (TESS; Ricker et al. 2015) is currently assembling sub-mmag precision space-based photometry of high-mass stars across the entire sky. This sample is yielding high quality observations of more massive stars than any homogeneous database to date (see e.g., Burssens et al. 2020; Labadie-Bartz et al. 2020). Characterization of the variability (or lack thereof) in these stars is providing the basis upon which predictions of physical mechanisms can be tested. In particular, stars that reveal coherent oscillation modes whose degree and azimuthal order can be identified, are suitable to be probeb via asteroseismology (Aerts 2021; Bowman 2020). So far, such detections and identifications are typically achieved in only about $10 \%$ of the observed OB-type stars, but this fraction is expected to increase thanks to TESS.

\section{DATA AVAILABILITY}

The K2 data presented in this paper were obtained from the Mikulski Archive for Space Telescopes (MAST) at the Space Telescope Science Institute (STScI), which is operated by the Association of Universities for Research in Astronomy, Inc., under NASA contract NAS5-26555. Support to MAST for these data is provided by the NASA Office of Space Science via grant NAG5-7584 and by other grants and contracts. Funding for the Kepler/K2 mission was provided by NASA's Science Mission Directorate. The HERMES data used within this article are available upon request. The binary modelling was carried out using ELLC (Maxted 2016) and the atmospheric modelling was carried out using FAstwind (Puls et al. 2005). The propagation of asymmetric uncertainties was performed using the SOAD code (Erdim \& Hüdaverdi 2019). The optimisation routines in this work made use of the NumPY (Harris et al. 2020) and EMCEE (Foreman-Mackey et al. 2013), and the plotting was carried out using MatPlotLiB (Hunter 2007). Mode identification results obtained with the software package FAMIAS developed in the framework of the FP6 European Coordination Action HELAS (http://www.helas-eu.org/).

\section{ACKNOWLEDGEMENTS}

We thank the referee for their comments which improved the manuscript. The research leading to these results has received funding from the European Research Council (ERC) under the European Union's Horizon 2020 research and innovation programme (grant agreement $\mathrm{N}^{\circ} 670519$ : MAMSIE and grant agreement $\mathrm{N}^{\circ} 772225$ : Multiples), from the KU Leuven Research Council (grant C16/18/005: PARADISE and C16/17/007: MAESTRO), 
from the Research Foundation Flanders (FWO) under grant agreements G0H5416N (ERC Runner Up Project) and G0A2917N (BlackGEM), from the FWO-Odysseus program under G0F8H6N, as well as from the BELgian federal Science Policy Office (BELSPO) through PRODEX grant PLATO. DMB gratefully acknowledges a senior postdoctoral fellowship from the Research Foundation Flanders (FWO) with grant agreement No. 1286521N. The computational resources and services used in this work were provided by the VSC (Flemish Supercomputer Center), funded by the Research Foundation - Flanders (FWO) and the Flemish Government - department EWI to PI Johnston.

\section{REFERENCES}

Abdul-Masih M., et al., 2019, ApJ, 880, 115

Aerts C., 2021, RMP, in press, 39, arXiv: 1912.12300

Aerts C., De Cat P., 2003, Space Sci. Rev., 105, 453

Aerts C., Rogers T. M., 2015, ApJ, 806, L33

Aerts C., de Pauw M., Waelkens C., 1992, A\&A, 266, 294

Aerts C., Puls J., Godart M., Dupret M. A., 2009, A\&A, 508, 409

Aerts C., Christensen-Dalsgaard J., Kurtz D. W., 2010, Asteroseismology,

Astronomy and Astrophysics Library. Springer-Verlag, Heidelberg

Aerts C., Simón-Díaz S., Groot P. J., Degroote P., 2014, A\&A, 569, A118

Aerts C., Van Reeth T., Tkachenko A., 2017, ApJ, 847, L7

Aerts C., et al., 2018, MNRAS, 476, 1234

Aerts C., Mathis S., Rogers T. M., 2019, ARA\&A, 57, 35

Aldoretta E. J., et al., 2015, AJ, 149, 26

Balona L. A., 1986, MNRAS, 219, 111

Balona L. A., Aerts C., Štefl S., 1999, MNRAS, 305, 519

Blomme R., et al., 2011, A\&A, 533, A4

Bonanos A. Z., Castro N., Macri L. M., Kudritzki R.-P., 2011, ApJ, 729, L9

Bowman D. M., 2017, Amplitude Modulation of Pulsation Modes in Delta Scuti Stars. Springer International Publishing, doi:10.1007/978-3-31966649-5

Bowman D. M., 2020, Frontiers in Astronomy and Space Sciences, 7, 70

Bowman D. M., et al., 2019a, Nature Astronomy, 3, 760

Bowman D. M., et al., 2019b, A\&A, 621, A135

Bowman D. M., Burssens S., Simón-Díaz S., Edelmann P. V. F., Rogers T. M., Horst L., Röpke F. K., Aerts C., 2020, A\&A, 640, A36

Breger M., et al., 1993, A\&A, 271, 482

Briquet M., Aerts C., 2003, A\&A, 398, 687

Briquet M., Morel T., Thoul A., Scuflaire R., Miglio A., Montalbán J., Dupret M. A., Aerts C., 2007, MNRAS, 381, 1482

Briquet M., et al., 2011, A\&A, 527, A112

Brott I., et al., 2011, A\&A, 530, A115

Burssens S., Bowman D. M., Aerts C., Pedersen M. G., Moravveji E., Buysschaert B., 2019, MNRAS, 489, 1304

Burssens S., et al., 2020, A\&A, 639, A81

Buysschaert B., et al., 2017, A\&A, 602, A91

Buysschaert B., Aerts C., Bowman D. M., Johnston C., Van Reeth T., Pedersen M. G., Mathis S., Neiner C., 2018, A\&A, 616, A148

Cantiello M., et al., 2009, A\&A, 499, 279

Charbonneau P., 1995, ApJS, 101, 309

Choi J., Dotter A., Conroy C., Cantiello M., Paxton B., Johnson B. D., 2016, ApJ, 823, 102

Claret A., Bloemen S., 2011, A\&A, 529, A75

Daszyńska-Daszkiewicz J., Walczak P., 2010, MNRAS, 403, 496

Daszyńska-Daszkiewicz J., Szewczuk W., Walczak P., 2013, MNRAS, 431, 3396

De Cat P., Aerts C., 2002, A\&A, 393, 965

De Cat P., De Ridder J., Hensberge H., Ilijic S., 2004, in Hilditch R. W., Hensberge H., Pavlovski K., eds, Astronomical Society of the Pacific Conference Series Vol. 318, Spectroscopically and Spatially Resolving the Components of the Close Binary Stars. pp 338-341

Degroote P., et al., 2009, A\&A, 506, 111

Degroote P., et al., 2010, Nature, 464, 259
Donati J. F., Semel M., Carter B. D., Rees D. E., Collier Cameron A., 1997, MNRAS, 291, 658

Edelmann P. V. F., Ratnasingam R. P., Pedersen M. G., Bowman D. M., Prat V., Rogers T. M., 2019, ApJ, 876, 4

Ekström S., et al., 2012, A\&A, 537, A146

Erdim M. K., Hüdaverdi M., 2019, in Turkish Physical Society 35th International Physics Congress (TPS35). p. 030023, doi:10.1063/1.5135421

Foreman-Mackey D., Hogg D. W., Lang D., Goodman J., 2013, PASP, 125, 306

Godart M., Simón-Díaz S., Herrero A., Dupret M. A., Grötsch-Noels A., Salmon S. J. A. J., Ventura P., 2017, A\&A, 597, A23

Gray D. F., 2005, The Observation and Analysis of Stellar Photospheres

Handler G., 2013, Asteroseismology. p. 207, doi:10.1007/978-94-007-5615$1 \_4$

Harris C. R., et al., 2020, Nature, 585, 357

Horst L., Edelmann P. V. F., Andrássy R., Röpke F. K., Bowman D. M., Aerts C., Ratnasingam R. P., 2020, A\&A, 641, A18

Howarth I. D., Siebert K. W., Hussain G. A. J., Prinja R. K., 1997, MNRAS, 284,265

Howell S. B., et al., 2014, PASP, 126, 398

Hunter J. D., 2007, Computing in Science \& Engineering, 9, 90

Ilijic S., Hensberge H., Pavlovski K., Freyhammer L. M., 2004, in Hilditch R. W., Hensberge H., Pavlovski K., eds, Astronomical Society of the Pacific Conference Series Vol. 318, Spectroscopically and Spatially Resolving the Components of the Close Binary Stars. pp 111-113

Johnston C., Buysschaert B., Tkachenko A., Aerts C., Neiner C., 2017, MNRAS, 469, L118

Johnston C., Tkachenko A., Aerts C., Molenberghs G., Bowman D. M., Pedersen M. G., Buysschaert B., Pápics P. I., 2019a, MNRAS, 482, 1231

Johnston C., Pavlovski K., Tkachenko A., 2019b, Astronomy and Astrophysics, 628, A25

Kiminki D. C., Kobulnicky H. A., 2012, ApJ, 751, 4

Kippenhahn R., Weigert A., Weiss A., 2012, Stellar Structure and Evolution. Springer-Verlag Berlin Heidelberg, doi:10.1007/978-3-642-30304-3

Koumpia E., Bonanos A. Z., 2012, A\&A, 547, A30

Krtička J., Feldmeier A., 2018, A\&A, 617, A121

Kupka F., Piskunov N., Ryabchikova T. A., Stempels H. C., Weiss W. W., 1999, A\&AS, 138, 119

Labadie-Bartz J., et al., 2020, AJ, 160, 32

Langer N., 2012, ARA\&A, 50, 107

Lehmann H., Tsymbal V., Mkrtichian D. E., Fraga L., 2006, A\&A, 457, 1033

Lohr M. E., Clark J. S., Najarro F., Patrick L. R., Crowther P. A., Evans C. J., 2018, A\&A, 617, A66

Maeder A., 2009, Physics, Formation and Evolution of Rotating Stars. Springer-Verlag Berlin Heidelberg, doi:10.1007/978-3-540-76949-1

Mahy L., et al., 2020a, A\&A, 634, A118

Mahy L., et al., 2020b, A\&A, 634, A119

Mantegazza L., Zerbi F. M., Sacchi A., 2000, A\&A, 354, 112

Martins F., Schaerer D., Hillier D. J., 2005, A\&A, 436, 1049

Mason B. D., Gies D. R., Hartkopf W. I., Bagnuolo William G. J., ten Brummelaar T., McAlister H. A., 1998, AJ, 115, 821

Maxted P. F. L., 2016, A\&A, 591, A111

Maxted P. F. L., et al., 2020, MNRAS, 498, 332

Mayer P., Harmanec P., Pavlovski K., 2013, A\&A, 550, A2

Mokiem M. R., de Koter A., Puls J., Herrero A., Najarro F., Villamariz M. R., 2005, A\&A, 441, 711

Moravveji E., Townsend R. H. D., Aerts C., Mathis S., 2016, ApJ, 823, 130

Otero S. A., 2007, Open European Journal on Variable Stars, 0072, 1

Pamyatnykh A. A., 1999, Acta Astron., 49, 119

Pápics P. I., et al., 2012, A\&A, 542, A55

Pavlovski K., Hensberge H., 2005, A\&A, 439, 309

Paxton B., et al., 2015, ApJS, 220, 15

Paxton B., et al., 2018, ApJS, 234, 34

Pedersen M. G., Aerts C., Pápics P. I., Rogers T. M., 2018, A\&A, 614, A128

Pope B. J. S., et al., 2019, ApJS, 245, 8

Prsa A., Matijevic G., Latkovic O., Vilardell F., Wils P., 2011, PHOEBE: 
PHysics Of Eclipsing BinariEs, Astrophysics Source Code Library (ascl:1106.002)

Prša A., et al., 2016, ApJS, 227, 29

Puls J., Urbaneja M. A., Venero R., Repolust T., Springmann U., Jokuthy A., Mokiem M. R., 2005, A\&A, 435, 669

Puls J., Vink J. S., Najarro F., 2008, A\&ARv, 16, 209

Raskin G., et al., 2011, A\&A, 526, A69

Ratnasingam R. P., Edelmann P. V. F., Rogers T. M., 2020, MNRAS, 497, 4231

Ricker G. R., et al., 2015, Journal of Astronomical Telescopes, Instruments, and Systems, 1, 014003

Rivinius T., Baade D., Štefl S., 2003, A\&A, 411, 229

Rogers T. M., McElwaine J. N., 2017, ApJ, 848, L1

Sana H., et al., 2012, Science, 337, 444

Sana H., et al., 2013, A\&A, 550, A107

Sana H., et al., 2014, ApJS, 215, 15

Sander A. A. C., Vink J. S., Hamann W. R., 2019, MNRAS, p. 2641

Schmid V. S., Aerts C., 2016, A\&A, 592, A116

Schrijvers C., Telting J. H., Aerts C., Ruymaekers E., Henrichs H. F., 1997, A\&AS, 121, 343

Schrijvers C., Telting J. H., Aerts C., 2004, A\&A, 416, 1069

Simón-Díaz S., Herrero A., 2014, A\&A, 562, A135

Simón-Díaz S., Godart M., Castro N., Herrero A., Aerts C., Puls J., Telting J., Grassitelli L., 2017, A\&A, 597, A22

Simón-Díaz S., Aerts C., Urbaneja M. A., Camacho I., Antoci V., Fredslund Andersen M., Grundahl F., Pallé P. L., 2018, A\&A, 612, A40

Southworth J., 2013, A\&A, 557, A119

Sundqvist J. O., Puls J., Feldmeier A., Owocki S. P., 2011, A\&A, 528, A64

Szewczuk W., Daszyńska-Daszkiewicz J., 2017, MNRAS, 469, 13

Tkachenko A., Van Reeth T., Tsymbal V., Aerts C., Kochukhov O., Debosscher J., 2013, A\&A, 560, A37

Tkachenko A., et al., 2020, A\&A, 637, A60

Torres G., Andersen J., Giménez A., 2010, ARA\&A, 18, 67

Uytterhoeven K., Telting J. H., Aerts C., Willems B., 2004, A\&A, 427, 593

Uytterhoeven K., Briquet M., Aerts C., Telting J. H., Harmanec P., Lefever K., Cuypers J., 2005, A\&A, 432, 955

Van Hamme W., Wilson R. E., 2007, ApJ, 661, 1129

Vink J. S., Muijres L. E., Anthonisse B., de Koter A., Gräfener G., Langer N., 2011, A\&A, 531, A132

Wade G. A., et al., 2016, MNRAS, 456, 2

Walczak P., et al., 2019, MNRAS, 485, 3544

White T. R., et al., 2017, MNRAS, 471, 2882

Wu T., Li Y., 2019, ApJ, 881, 86

Zima W., 2006, A\&A, 455, 227

Zima W., 2008, Communications in Asteroseismology, 157, 387

Zima W., et al., 2006, A\&A, 455, 235

de Mink S. E., Langer N., Izzard R. G., Sana H., de Koter A., 2013, ApJ, 764,166

\section{APPENDIX A: MOMENT METHOD PERIODOGRAMS}

This paper has been typeset from a $\mathrm{T}_{\mathrm{E}} \mathrm{X} / \mathrm{LAT}_{\mathrm{E}} \mathrm{X}$ file prepared by the author.

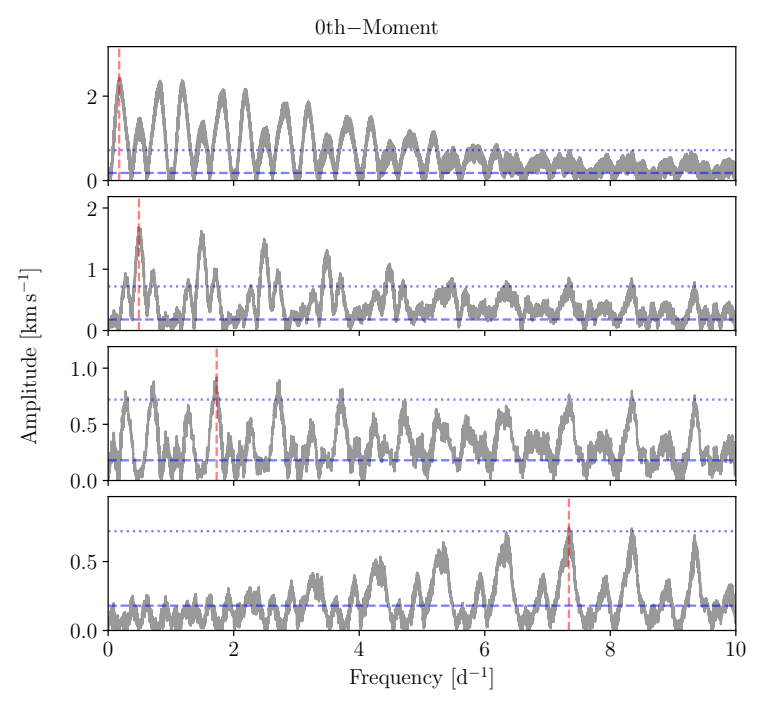

Figure A1. Periodograms of the 0th-moment of the LSD profiles. Extracted frequencies denoted by red vertical line. Dashed blue lines show noise level of pre-whitened data set, dotted blue lines denote four times the noise level.

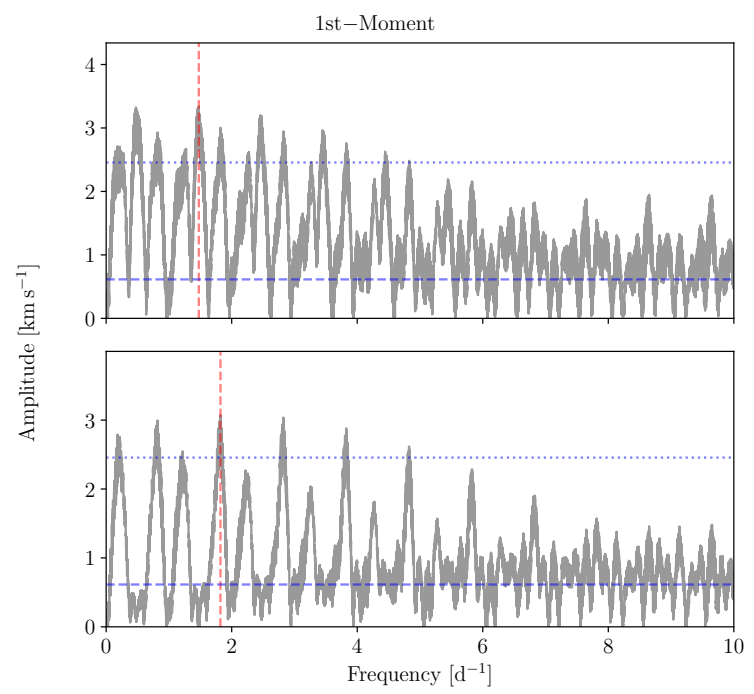

Figure A2. Same as Fig. A1 but for 1st moment. 
2nd-Moment

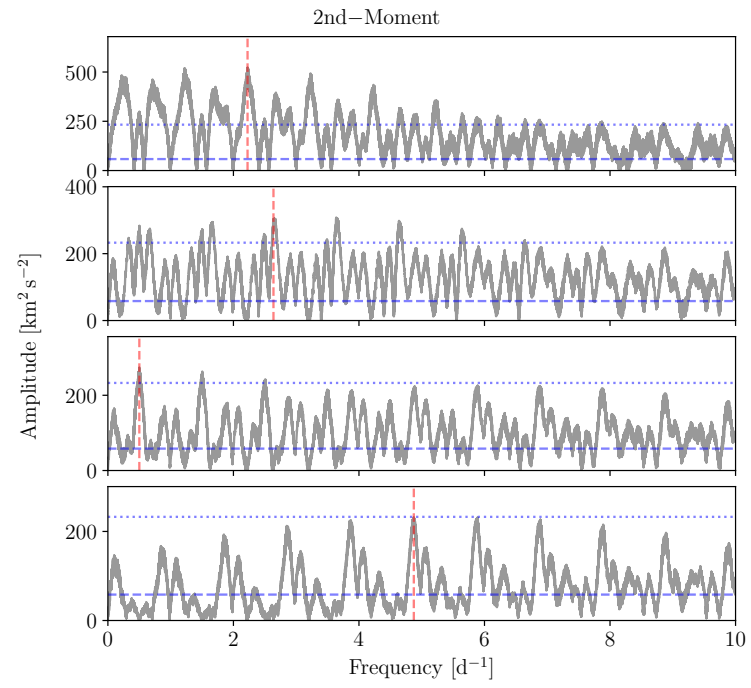

Figure A3. Same as Fig. A1 but for 2nd moment.

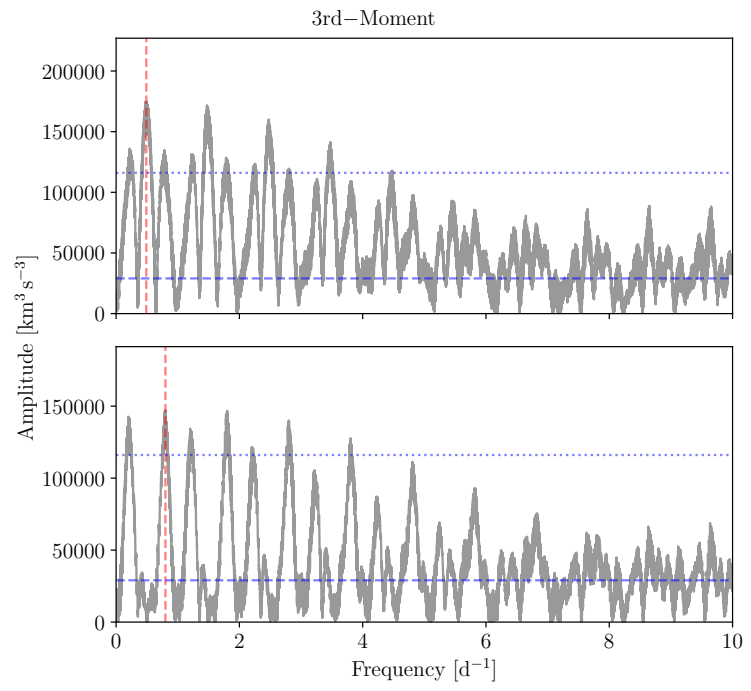

Figure A4. Same as Fig. A1 but for 3rd moment. 